\title{
6D F-theory models and elliptically fibered Calabi-Yau threefolds over semi-toric base surfaces
}

\section{Gabriella Martini and Washington Taylor}

Center for Theoretical Physics, Department of Physics, Massachusetts Institute of Technology, 77 Massachusetts Avenue, Cambridge, MA 02139, U.S.A.

E-mail: gmartini@mit.edu, wati@mit.edu

ABSTRACT: We carry out a systematic study of a class of 6D F-theory models and associated Calabi-Yau threefolds that are constructed using base surfaces with a generalization of toric structure. In particular, we determine all smooth surfaces with a structure invariant under a single $\mathbb{C}^{*}$ action (sometimes called "T-varieties" in the mathematical literature) that can act as bases for an elliptic fibration with section of a Calabi-Yau threefold. We identify 162,404 distinct bases, which include as a subset the previously studied set of strictly toric bases. Calabi-Yau threefolds constructed in this fashion include examples with previously unknown Hodge numbers. There are also bases over which the generic elliptic fibration has a Mordell-Weil group of sections with nonzero rank, corresponding to non-Higgsable U(1) factors in the 6D supergravity model; this type of structure does not arise for generic elliptic fibrations in the purely toric context.

KEYwords: F-Theory, Differential and Algebraic Geometry, Supergravity Models

ARXIV EPRINT: 1404.6300 


\section{Contents}

1 Introduction 1

$2 \quad \mathbb{C}^{*}$-surfaces and F-theory vacua $\quad 3$

2.1 6D F-theory models 3

2.2 General classification of 6D F-theory base surfaces 3

2.3 Toric base surfaces 5

$2.4 \mathbb{C}^{*}$-base surfaces $\quad 7$

2.5 Curves of self-intersection $-9,-10$, and $-11 \quad 8$

3 Enumeration of bases $\quad 9$

3.1 Classification and enumeration of $\mathbb{C}^{*}$-bases 9

$\begin{array}{lll}3.2 & \text { Distribution of bases } & 10\end{array}$

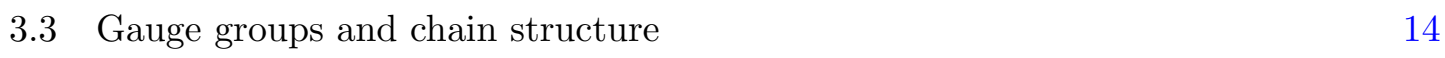

4 Calabi-Yau geometry $\quad 16$

$\begin{array}{lll}\text { 4.1 Calabi-Yau geometry of elliptic fibrations over } \mathbb{C}^{*} \text {-bases } & 17\end{array}$

$\begin{array}{lll}4.2 & \text { Counting neutral hypermultiplets } & 18\end{array}$

4.3 Distribution of Hodge numbers 22

4.4 Redundancies from -2 clusters 23

4.5 Calabi-Yau threefolds with new Hodge numbers 26

4.6 Calabi-Yau threefolds with nontrivial Mordell-Weil rank 26

$\begin{array}{llr}5 & \text { Conclusions } & 28\end{array}$

A Rules for connecting clusters in $\mathbb{C}^{*}$-bases $\quad 30$

B Bases giving Calabi-Yau threefolds with new Hodge numbers 32

$\begin{array}{ll}\text { C Bases giving } \mathrm{U}(1) \text { factors } & 33\end{array}$

\section{Introduction}

Since the early days of string theory, much effort has been devoted to understanding the geometry of string compactifications. Calabi-Yau threefolds are one of the most central and best-studied classes of compactification geometries. These manifolds can be used to compactify ten-dimensional superstring theories to give four-dimensional supersymmetric theories of gravity and gauge fields [1, 2]. Calabi-Yau threefolds that admit an elliptic fibration with section can also be used to compactify F-theory to give six-dimensional supergravity theories [3-5]. 
While mathematicians and physicists have used many methods to construct and study Calabi-Yau threefolds (see [6] for a recent review), one of the main approaches that has been fruitful for systematically classifying large numbers of Calabi-Yau geometries is the mathematical framework of toric geometry [7]. The power of toric geometry is that many geometric features of a space are captured in a simple combinatorial framework that lends itself to straightforward calculations for many quantities of interest. An approach was developed by Batyrev [8] for describing Calabi-Yau manifolds as hypersurfaces in toric varieties in terms of the combinatorics of reflexive polytopes. Kreuzer and Skarke have identified some 473.8 million four-dimensional reflexive polytopes that can be used to construct Calabi-Yau threefolds in this way [9].

In this paper, following [10], we study a class of spaces that is more general than the set of toric varieties, but retains some of the combinatorial simplicity of toric geometry. This allows us to construct a large class of elliptically fibered Calabi-Yau threefolds that need not have any realization in a strictly toric language. In particular, we focus on complex surfaces that admit at least one $\mathbb{C}^{*}$ action but not necessarily the action of a product $\mathbb{C}^{*} \times \mathbb{C}^{*}$ needed for a full toric structure. Complex algebraic varieties of dimension $n$ that admit an effective action of $\left(\mathbb{C}^{*}\right)^{k}$ are known in the mathematical literature as "T-varieties". In this paper we simply use the term " $\mathbb{C}^{*}$-surface" to denote a surface with a $\mathbb{C}^{*}$ action, in part to avoid confusion with the plethora of "T-" objects already filling the string theory literature ("T-duality", "T-folds", "T-branes", etc.) A review of some of the mathematical results and literature on more general T-varieties can be found in [11].

The primary focus of this paper is the systematic classification and study of all smooth $\mathbb{C}^{*}$-surfaces that can act as bases for an elliptic fibration with section where the total space is a Calabi-Yau threefold. We use these surfaces to compactify F-theory to six dimensions. The close correspondence between the geometry of the F-theory compactification surface and the physics of the associated 6D supergravity theory provides a powerful tool for understanding both geometry and physics. A general characterization of smooth base surfaces $^{1}$ (not necessarily toric or $\mathbb{C}^{*}$ ) was given in [10]. The basic idea is that any base can be classified by the intersection structure of effective irreducible divisors of selfintersection -2 or below. The intersection structure of the base directly corresponds to the generic nonabelian gauge group in the "maximally Higgsed" 6D supergravity theory from an F-theory construction. There are strong constraints on the intersection structures that can arise; these constraints made possible an explicit enumeration of all toric base surfaces in [12], and the geometry of the associated Calabi-Yau threefolds was explored in [13]. In this paper we construct and enumerate the more general class of $\mathbb{C}^{*}$-bases and explore the corresponding Calabi-Yau geometries. In particular, we identify some (apparently) new Calabi-Yau threefolds including some threefolds with novel properties.

In section 2 we describe the class of $\mathbb{C}^{*}$-base surfaces. We summarize the results of the complete enumeration of these bases in section 3. The corresponding Calabi-Yau threefolds, including some models with interesting new features, are described in section 4 . Section 5 contains concluding remarks. Appendices A-C contain tables of useful data.

\footnotetext{
${ }^{1}$ Throughout this paper we use the term "base surface" as shorthand for "surface that can act as the base of an elliptically-fibered Calabi-Yau threefold with section".
} 


\section{$2 \quad \mathbb{C}^{*}$-surfaces and F-theory vacua}

We begin by briefly summarizing the results of $[10,12]$. The methods used here are closely related to those developed in these papers.

\section{$2.1 \quad 6 D$ F-theory models}

A 6D F-theory model is defined by a Calabi-Yau threefold that is an elliptic fibration (with section) over a complex base surface $B$. The structure of the resulting $6 \mathrm{D}$ supergravity theory is determined by the geometry of $B$ and the elliptic fibration [4,5]. In particular, the number of tensor multiplets $T$ in the $6 \mathrm{D}$ theory is related to the topology of the base $B$ through $T=h^{1,1}(B)-1$. The elliptic fibration can be described by a Weierstrass model over $B$

$$
y^{2}=x^{3}+f x+g,
$$

where $f, g$ are sections of the line bundles $\mathcal{O}(-4 K), \mathcal{O}(-6 K)$, with $K$ the canonical class of $B$. Codimension one vanishing loci of $f, g$, and the discriminant locus $\Delta=4 f^{3}+27 g^{2}$, where the elliptic fibration becomes singular, give rise to vector multiplets for a nonabelian gauge group in the $6 \mathrm{D}$ theory. Codimension two vanishing loci give matter in the $6 \mathrm{D}$ theory; the matter lives in a representation of the nonabelian gauge group that is determined by the geometry. For more detailed background regarding the relation of $6 \mathrm{D}$ physics to $\mathrm{F}$ theory geometry, see the reviews [14-16]; a recent systematic analysis of these 6D models from the M-theory point of view appears in [17]).

\subsection{General classification of 6D F-theory base surfaces}

A general approach to systematically classifying base surfaces $B$ for $6 \mathrm{D}$ F-theory compactifications was developed in [10]. This approach is based on identifying irreducible components in the structure of effective divisors on $B$, composed of intersecting combinations of curves of negative self-intersection over which the generic elliptic fibration is singular. Each such irreducible component corresponds to a "non-Higgsable cluster" of gauge algebra summands and (in some cases) charged matter that appears in the 6D supergravity model arising from an F-theory compactification on a generic elliptic fibration over $B$; the term "non-Higgsable cluster" (which we sometimes abbreviate as "cluster") refers to the fact that for any such configuration there are no matter fields that can lead to a Higgsing of the corresponding nonabelian gauge group. For example, a single irreducible effective curve in $B$ with self-intersection -4 corresponds to an $\mathfrak{s o}(8)$ term in the gauge algebra with no charged matter. The simplest example of this occurs for F-theory on the Hirzebruch surface $\mathbb{F}_{4}[4,5]$. There are strong geometric constraints (discussed further below) on the configurations of curves that can live in a base supporting an elliptic fibration. The set of possible non-Higgsable clusters is thus rather small. A complete list is given in table 1 , and depicted in figure 1. These clusters are described by configurations of curves having self-intersection -2 or less, with one curve having self-intersection -3 or below. General configurations of -2 curves with no curves of self-intersection -3 or below are also possible. These clusters carry no gauge group, and generally represent limiting points of 


\begin{tabular}{|c|c|c|}
\hline Cluster & gauge algebra & $H_{\text {charged }}$ \\
\hline$(-12)$ & $\mathfrak{e}_{8}$ & 0 \\
$(-8)$ & $\mathfrak{e}_{7}$ & 0 \\
$(-7)$ & $\mathfrak{e}_{7}$ & 28 \\
$(-6)$ & $\mathfrak{e}_{6}$ & 0 \\
$(-5)$ & $\mathfrak{f}_{4}$ & 0 \\
$(-4)$ & $\mathfrak{s o}(8)$ & 0 \\
$(-3,-2,-2)$ & $\mathfrak{g}_{2} \oplus \mathfrak{s u}(2)$ & 8 \\
$(-3,-2)$ & $\mathfrak{g}_{2} \oplus \mathfrak{s u}(2)$ & 8 \\
$(-3)$ & $\mathfrak{s u}(3)$ & 0 \\
$(-2,-3,-2)$ & $\mathfrak{s u}(2) \oplus \mathfrak{s o}(7) \oplus \mathfrak{s u}(2)$ & 16 \\
$(-2,-2, \ldots,-2)$ & no gauge group & 0 \\
\hline
\end{tabular}

Table 1. Allowed "non-Higgsable clusters" of irreducible effective divisors with self-intersection -2 or below, and corresponding contributions to the gauge algebra and matter content of the $6 \mathrm{D}$ theory associated with F-theory compactifications on a generic elliptic fibration (with section) over a base containing each cluster.

$$
\begin{gathered}
-m \in \\
\{-3,-4, \ldots,-8,-12\}
\end{gathered}
$$$$
\mathfrak{s u}(3), \mathfrak{s o}(8), \mathfrak{f}_{4}
$$$$
\mathfrak{e}_{6}, \mathfrak{e}_{7}, \mathfrak{e}_{8}
$$

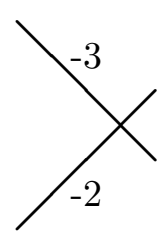

$\mathfrak{g}_{2} \oplus \mathfrak{s u}(2)$

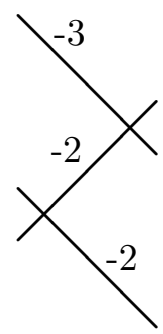

$\mathfrak{g}_{2} \oplus \mathfrak{s u}(2)$

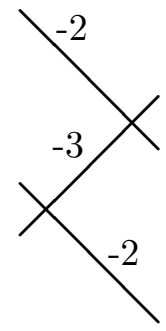

$\mathfrak{s u}(2) \oplus \mathfrak{s o}(7) \oplus \mathfrak{s u}(2)$

Figure 1. Clusters of intersecting curves that must carry a nonabelian gauge group factor. For each cluster the corresponding gauge algebra is noted and the gauge algebra and number of charged matter hypermultiplet are listed in table 1 .

bases without these -2 curves; for example, the Hirzebruch surface $\mathbb{F}_{2}$ contains a single isolated -2 curve, and is a limit of the surface $\mathbb{F}_{0}$.

The restriction on the types of allowed clusters comes from the constraint that $f, g$ cannot vanish to degrees 4,6 respectively on any curve or at any point in $B$. If the degree of vanishing is too high on a curve, there is no way to construct a Calabi-Yau from the elliptic fibration. If the vanishing is too high at a point, the point must be blown up to form another base $B^{\prime}$ that supports a Calabi-Yau. The vanishing degrees of $f, g$ on a curve can be determined from the Zariski decomposition of $-n K$ : using the fact that any effective divisor $A$ that has a negative intersection with an effective irreducible $B$ having negative self-intersection must contain $B$ as a component $(A \cdot B<0, B \cdot B<0 \Rightarrow A=B+C$ with $C$ effective), the degree of vanishing of $A=-4 K,-6 K,-12 K$ on any irreducible effective 
curve or combination of curves can be computed; the degree of vanishing at a point where two curves intersect is simply the sum of the degrees of vanishing on the curves. The details of this computation for general combinations of intersecting divisors are worked out in [10].

All effective irreducible curves of self-intersection -2 or below in the base appear in the clusters described above. Furthermore, again because of restrictions on the degrees of $f, g$ on curves and intersection points, only certain combinations of the allowed clusters can be connected by -1 curves in $B$. For example, if a -1 curve intersects a -12 curve, then the -1 curve cannot intersect any other curve contained in a non-Higgsable cluster except a -2 curve contained in a $(-2,-2,-3)$ cluster. A complete table of the possible combinations of clusters that can be connected by a -1 curve is given in [10]. The part of that table that is relevant for this paper is reproduced in appendix A.

For any base surface $B$, the structure of non-Higgsable clusters determines the minimal nonabelian gauge group and matter content of the $6 \mathrm{D}$ supergravity theory corresponding to a generic elliptic fibration with section over $B$. For each distinct base $B$ there can be a wide range of models with different nonabelian gauge groups, which can be realized by tuning the Weierstrass model to increase the degrees of $f, g$ over various divisors. For example, for the simplest base surface $B=\mathbb{P}^{2}$, there are thousands of branches of the theory with different nonabelian gauge group and matter content, some of which are explored in $[18,19]$. Each of these branches corresponds to a different Calabi-Yau threefold after the singularities associated with the nonabelian gauge group factors are resolved. But for each of these models, by maximally Higgsing matter fields in the supergravity theory the gauge group can be completely broken and the geometry becomes that of a generic elliptic fibration over $\mathbb{P}^{2}$. Focusing on the base surface $B$ dramatically simplifies the problem of classifying F-theory compactifications and elliptically fibered Calabi-Yau threefolds, by removing the additional complexity associated with the details of specific Weierstrass models and associated fibrations.

The mathematics of minimal surface theory [20,21] gives a simple picture of how the set of allowed base surfaces $B$ are connected, unifying the space of $6 \mathrm{D}$ supergravity theories that arise from F-theory. All smooth bases ${ }^{2} B$ for $6 \mathrm{D}$ F-theory models can be constructed by blowing up a finite set of points on one of the minimal bases $\mathbb{F}_{m}(0 \leq m \leq 12, m \neq 1)$ or $\mathbb{P}^{2}[22]$. The number of distinct topological types for $B$ is finite [23, 24]. In principle, all smooth bases $B$ can be systematically constructed by successively blowing up points on the minimal bases allowing only the clusters of irreducible divisors from table 1. In [12], the complete set of toric bases was constructed in this fashion, and in this paper we carry out the analogous construction for $\mathbb{C}^{*}$-bases.

\subsection{Toric base surfaces}

The set of toric base surfaces form a subset of the more general class of $\mathbb{C}^{*}$-bases that we focus on in this paper. The structure of toric bases is relatively simple and provides a useful foundation for our analysis of $\mathbb{C}^{*}$-bases.

\footnotetext{
${ }^{2}$ aside from the Enriques surface, which gives rise to a simple $6 \mathrm{D}$ model with no gauge group or matter and is connected in a more complicated way to the branches associated with other bases.
} 


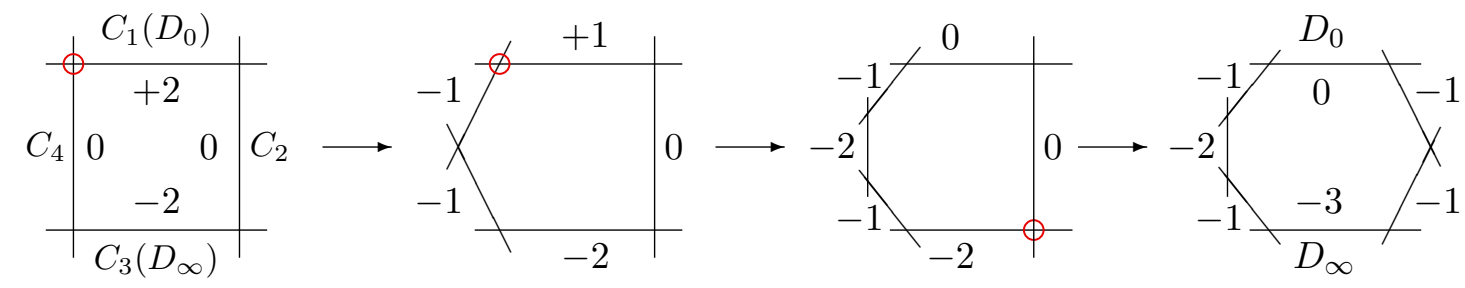

Figure 2. Toric base surfaces for 6D F-theory models produced by blowing up a sequence of points on $\mathbb{F}_{2}$.

A toric surface can be described alternatively in terms of the toric fan or in terms of the set of toric effective divisors. The fan for a compact toric surface is defined [7] by a sequence of vectors $v_{1}, \ldots, v_{k} \in N=\mathbb{Z}^{2}$ defining $1 \mathrm{D}$ cones, or rays, along with $2 \mathrm{D}$ cones spanned by vectors $v_{i}, v_{i+1}$ (including a $2 \mathrm{D}$ cone spanned by $v_{k}, v_{1}$; all related conditions include this periodicity though we do not repeat this explicitly in each case), and the 0D cone at the origin. The origin represents the torus $\left(\mathbb{C}^{*}\right)^{2}$, while the $1 \mathrm{D}$ rays represent divisors and the $2 \mathrm{D}$ cones represent points in the compact toric surface. The surface is smooth if the rays $v_{i}, v_{i+1}$ defining each $2 \mathrm{D}$ cone span a unit cell in the lattice. The irreducible effective toric divisors are a set of curves $^{3} C_{i}, i=1, \ldots, k$, associated with the vectors $v_{i}$. The divisors have self-intersection $C_{i} \cdot C_{i}=n_{i}$, where $-n v_{i}=v_{i-1}+v_{i+1}$, and nonvanishing pairwise intersections $C_{i} \cdot C_{i+1}=C_{k} \cdot C_{1}=1$. These divisors can be depicted graphically as a loop (See figure 2). The sets of consecutive divisors of self-intersection $n_{i} \leq-2$ in the loop are constrained by the cluster analysis of [10] to contain only the sequences (with either orientation) $(-3,-2),(-3,-2,-2),(-2,-3,-2),(-m)$, with $3 \leq m \leq 12$, and $(-2, \ldots,-2)$ with any number of -2 curves.

All smooth toric base surfaces (aside from $\mathbb{P}^{2}$ ) can be constructed by starting with a Hirzebruch surface $\mathbb{F}_{m}$ that is associated with the divisor self-intersection sequence $\left[n_{1}, n_{2}, n_{3}, n_{4}\right]=[m, 0,-m, 0]\left(v_{1}=(0,1), v_{2}=(1,0), v_{3}=(0,-1), v_{4}=(-1,-m)\right)$, and blowing up a sequence of intersection points between adjacent divisors. Blowing up the intersection point between divisors $C_{i}$ and $C_{i+1}$ gives a new toric base with a -1 curve inserted between these divisors, and self-intersections of the previously intersecting divisors each reduced by one (See figure 2). Such blow-ups are the only ones possible that maintain the toric structure by preserving the action of $\left(\mathbb{C}^{*}\right)^{2}$ on the base. Each Hirzebruch surface can be viewed as a $\mathbb{P}^{1}$ bundle over $\mathbb{P}^{1}$. The divisors of self-intersection $\pm m$ can be viewed as sections $\Sigma_{ \pm}$of this bundle, which in a local coordinate chart $(z, w) \in \mathbb{C} \times \mathbb{C}$ are at the points $(z, 0),(z, \infty)$ in the fibers, while the divisors of self-intersection 0 can be viewed as fibers $(0, w)$ and $(\infty, w)$. All points that can be blown up while maintaining the toric structure are located at the points invariant under the $\left(\mathbb{C}^{*}\right)^{2}$ action: $(0,0),(0, \infty),(\infty, 0),(\infty, \infty)$ (or in exceptional divisors produced when these points are blown up). In particular, any

\footnotetext{
${ }^{3}$ Note that the notation and ordering used here for curves and associated rays in a toric base differs from that used in [12].
} 
smooth toric base surface that supports an elliptically fibered Calabi-Yau threefold has a description in terms of a closed loop of divisors containing divisors $D_{0}, D_{\infty}$ associated with the divisors $C_{1}, C_{3}$ in the original Hirzebruch surface (these are the sections $\Sigma_{ \pm}$) and two linear chains of divisors connecting $D_{0}$ to $D_{\infty}$. These chains are formed from the divisors $C_{2}$ and $C_{4}$ in the original Hirzebruch surface, along with all exceptional divisors from blowing up points on these original curves. This is illustrated in figure 2 .

Note that some smooth toric base surfaces can be formed in different ways from blowups of Hirzebruch surfaces, so that different divisors $D_{0}, D_{\infty}$ play the role of the sections $\Sigma_{ \pm}$. In enumerating all toric base surfaces, such duplicates must be eliminated by considering equivalences of the loops of self-intersection numbers up to rotation and reflection.

\section{$2.4 \mathbb{C}^{*}$-base surfaces}

A more general class of bases $B$ was described in [12]. Generalizing the toric construction by allowing blow-ups at arbitrary points $(z, 0),(z, \infty)$ can give rise to an arbitrary number of fibers containing curves of negative self-intersection, while maintaining the $\mathbb{C}^{*}$ action $\left(1 \times \mathbb{C}^{*}\right)$ that leaves the sections $\Sigma_{ \pm}$invariant. The resulting structure is closely analogous to that of the toric bases described above, except that there can be more than two chains of intersecting divisors connecting $D_{0}, D_{\infty}$, associated with distinct blown-up fibers of the original Hirzebruch surface. Graphically, the intersection structure of effective irreducible divisors for such a base can be depicted as the pair of horizontal divisors $D_{0}, D_{\infty}$ (associated with $\Sigma_{ \pm}$in the original Hirzebruch surface), along with an arbitrary number $N$ of chains of divisors $D_{i, j}, i=1, \ldots, N$ connecting $D_{0}, D_{\infty}$. The $i$ th chain is realized by blowing up a fiber in $\mathbb{F}_{m}$, and contains divisors $D_{i, j}, j=1, \ldots, k_{i}$, with self-intersections and adjacent intersections as described above $\left(D_{i, j} \cdot D_{i, j}=n_{i, j}<0, D_{i, j} \cdot D_{i, j+1}=1\right)$ except that $D_{i, 1} \cdot D_{0}=1, D_{i, k_{i}} \cdot D_{\infty}=1$, and $D_{i, 1} \cdot D_{i, k_{i}}=0$ unless $k_{i}=2$. (See figure 3.) We refer to bases of this form as $\mathbb{C}^{*}$-bases. In this language, the toric bases correspond to those $\mathbb{C}^{*}$-bases with $N=2$ (or fewer) chains. The divisor intersection structure of a $\mathbb{C}^{*}$-base is constrained by the set of allowed clusters found in [10] in a similar way to toric bases, but with additional possibilities associated with the existence of multiple connections to the divisors $D_{0}, D_{\infty}$ that provide branchings in the intersection structure. For example, if $D_{0}$ has self-intersection $D_{0} \cdot D_{0}=-3$, and is connected to chains with self-intersections $\left(n_{1,1}, n_{1,2}, \ldots\right)=(-2,-1, \ldots),\left(n_{2,1}, n_{2,2}, \ldots\right)=(-2,-1, \ldots)$, then any further chains $i>2$ must satisfy $n_{i, 1}=-1, n_{i, 2} \geq-4$. The complete set of constraints on how divisors can be connected is described in appendix A, including some additional constraints beyond those described in [10] related to branchings on curves of self-intersection $-n<-1$.

Note that the value of $T=h^{1,1}(B)-1$ can be determined directly from the intersection structure of a $\mathbb{C}^{*}$-base. Each blow-up adds one to $T$. Each blow-up along a new fiber increases $N$ by one and creates a new $(-1,-1)$ chain with $k_{N}=2$, while each blow-up on an intersection in chain $i$ increases $k_{i}$ by 1 . Matching with the Hirzebruch surfaces, which have $N=0, T=1$, we have

$$
T=h^{1,1}(B)-1=\left(\sum_{i=1}^{N} k_{i}\right)-N+1 .
$$




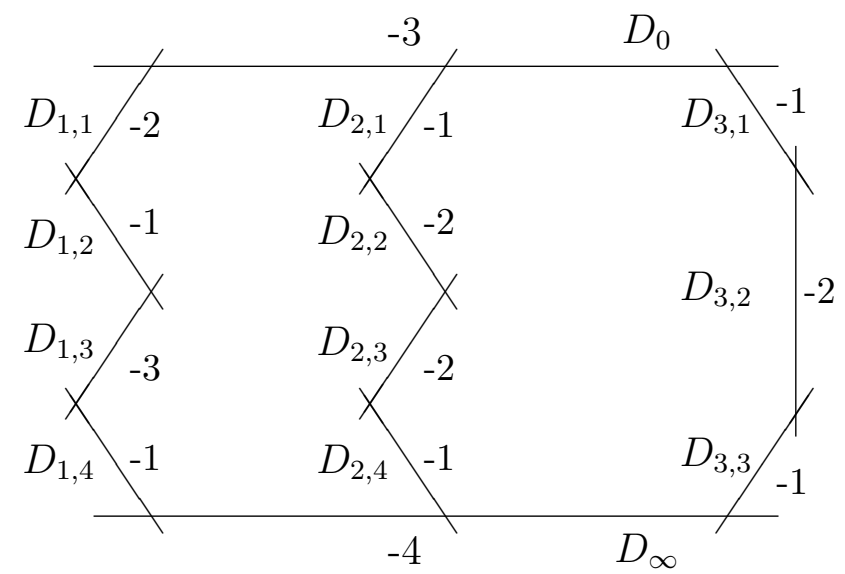

Figure 3. A $\mathbb{C}^{*}$-base $B$ is characterized by irreducible effective divisors $D_{0}, D_{\infty}$ connected by any number of linear chains of divisors $D_{i, j}$ with intersections obeying the cluster rules of [10]. All such bases can be realized as multiple blow-ups of a Hirzebruch surface $\mathbb{F}_{m}$ that preserve the action of a single $\mathbb{C}^{*}$ on $B$.

\subsection{Curves of self-intersection $-9,-10$, and -11}

There is one further issue that arises in systematically classifying toric and/or $\mathbb{C}^{*}$-bases. As shown in [10], for any base containing a curve $C$ of self-intersection $-9,-10$, or -11 , there is a point on $C$ where the Weierstrass functions $f, g$ vanish to degrees 4,6 , so that point in the base must be blown up for the base to support an elliptically fibered Calabi-Yau threefold. If the curve $C$ is a divisor associated with a section, $D_{0}$ or $D_{\infty}$, then blowing up this point simply adds another $(-1,-1)$ chain to the $\mathbb{C}^{*}$-surface (figure 4 ). If $C$ is an element of one of the chains, however, then blowing up the point on $C$ generally takes the base out of the class of $\mathbb{C}^{*}$ - or toric surfaces.

For strictly toric or $\mathbb{C}^{*}$-surfaces, therefore, we should not include any bases containing curves of self-intersection $-9,-10$, or -11 . For several reasons, however, we find it of interest to include bases with such curves even when the blow-up leaves the context of $\mathbb{C}^{*}$-surfaces. In particular, we are interested in exploring the widest range of bases possible that can be systematically analyzed. Thus, while the bases arising from blowing up -9, -10, or 11 curves on connecting chains generally takes us outside the $\mathbb{C}^{*}$ context we have done a complete enumeration of surfaces including these additional types of curves (which we refer to as "not-strictly $\mathbb{C}^{*}$-bases," or "NSC-bases" for short), with the understanding that the blown-up smooth surfaces that support elliptic Calabi-Yau fibrations are no longer strictly $\mathbb{C}^{*}$-surfaces. One strong argument in favor of including these bases in our analysis is that the base with the largest known value of $h^{1,1}(B)=491$, corresponding to the $6 \mathrm{D}$ F-theory compactification with the largest gauge group, is of this type. This geometry was first identified in $[25,26]$, and was studied further in [12, 13]. This geometry arises from a toric base that contains two -11 curves that cannot be associated with $D_{0, \infty}$, so the 


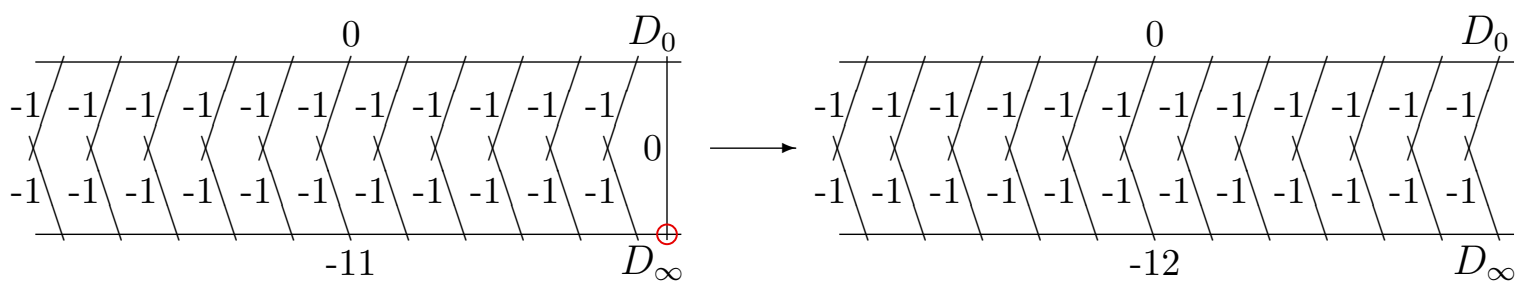

Figure 4. The $\mathbb{C}^{*}$-base with $N=11, n_{0}=0, n_{\infty}=-11$ and $11(-1,-1)$ chains has a singular point on $D_{\infty}$ where the base must be blown up, giving the smooth base with $N=12, n_{\infty}=-12$, and $12(-1,-1)$ chains.

blown-up smooth base surface is neither strictly toric nor $\mathbb{C}^{*}$. We included toric bases with $-9,-10,-11$ curves in [12]. In the next section we discuss how these are systematically included in the enumeration of $\mathbb{C}^{*}$-bases in the analysis of this paper.

For (NSC) surfaces that contain $-9,-10$, or -11 curves, an additional contribution to $T$ must be added for each blow-up needed to reach a smooth surface with only allowed clusters (i.e., with -12 curves instead), so the equation for $T$ is modified from (2.2) to

$$
T=h^{1,1}(B)-1=\left(\sum_{i=1}^{N} k_{i}\right)-N+c_{11}+2 c_{10}+3 c_{9}+1,
$$

where $c_{k}$ is the number of curves with self-intersection $-k$.

\section{Enumeration of bases}

We now summarize the results of a full enumeration of the set of $\mathbb{C}^{*}$-base surfaces relevant for F-theory compactification. In this section we describe the features relevant for the corresponding 6D supergravity theories. The following section (section 4) focuses on aspects of the associated Calabi-Yau geometries.

\subsection{Classification and enumeration of $\mathbb{C}^{*}$-bases}

Any $\mathbb{C}^{*}$-surface can be characterized by the self-intersection numbers $n_{0}, n_{\infty}$ of the divisors $D_{0}, D_{\infty}$ associated with the sections $\Sigma_{ \pm}$, the number $N$ of chains associated with distinct fibers along which blow-ups have occurred, and the intersection structure of the divisors connected along each chain, characterized by the integers $n_{i, j}$ as described above. Thinking of each base $B$ as a blow-up of $\mathbb{F}_{m}$, each chain is constructed by first blowing up a point at the intersection of a fiber $F_{i}$ in $\mathbb{F}_{m}$ with either $D_{0}$ or $D_{\infty}$, giving a chain of length 2 containing a pair of intersecting -1 curves. Additional blow-ups can then be performed at the intersections either between pairs of adjacent divisors along the chain, or between the divisors at the end of the chain and $D_{0}$ or $D_{\infty}$. Each nontrivial chain thus is associated 
with a decrease in the self-intersection of $D_{0}$ or $D_{\infty}$ by at least one. Since the minimum values of $n_{0}, n_{\infty}$ are -12 (as determined by the allowed non-Higgsable clusters), and their associated intersection numbers for $\Sigma_{ \pm}$on $\mathbb{F}_{m}$ are equal and opposite, the maximum number of possible nontrivial chains is $N=24$. Constructions with $N=2$ or fewer nontrivial chains are realized already in the toric context described in [12]; we have included these cases in the complete analysis here, though the counting is slightly different due to the treatment of $-9,-10$, and -11 curves.

To enumerate all possible $\mathbb{C}^{*}$-bases then, we can proceed by first constructing all possible nontrivial chains associated with at most 24 blow-ups of points on the ending divisors of each chain (similar constructions were described in $[12,27]$ ). We then consider all possible combinations of these chains compatible with the bound on intersection numbers of the sections $D_{0, \infty}$. To implement this enumeration algorithmically, we consider the set of partitions of $k$ such that $1 \leq k \leq 24$, which are equivalent to all valid combinations for the number of blow-ups at the intersection of distinct fibers with $D_{0}$ or $D_{\infty}$ for any $\mathbb{F}_{m}$. Then for each $\mathbb{F}_{m}$ and partition $\lambda \vdash k$ such that $k=k_{1}+\ldots+k_{N}$, we can identify all combinations of chains associated with $k_{1}, \ldots, k_{N}$ blow-ups on the ending divisors such that the intersection of these chains with $D_{0}$ and $D_{\infty}$ collectively satisfy the F-theory rules contained in table 1 and table 3 . Doing so for every achievable combination of $n_{0}, n_{\infty}$ with $\lambda \vdash k$ gives a systematic method for enumerating all valid $\mathbb{C}^{*}$-bases.

We have carried out the complete enumeration of all strictly $\mathbb{C}^{*}$-bases (i.e., including no $-9,-10,-11$ curves) for all values of $N$ from 0 to 24 . We have also enumerated those bases which are not strictly $\mathbb{C}^{*}$ (or toric), due to $-9,-10$, or -11 curves on the fiber chains. We have not considered bases with $-9,-10$, or -11 curves for $D_{0}$ or $D_{\infty}$, since blowing up these curves gives a $\mathbb{C}^{*}$-base with larger $N$, so including these would simply amount to overcounting. The cases $N=0,1,2$ represent toric bases. We have not included toric bases with $-9,-10,-11$ curves on fiber chains when there is an equivalent toric base where such a curve can be mapped to $D_{0}$ or $D_{\infty}$ by a rotation of the loop of toric divisors. These cases are already counted elsewhere in the set of $\mathbb{C}^{*}$ bases, as discussed in more detail in the following section.

\subsection{Distribution of bases}

The number of distinct bases, uniquely determined by $N, n_{0}, n_{\infty}$, and the intersection configuration of the curves on the $N$ chains (up to the symmetries associated with permutations of the chains and the simultaneous reflection of all chains combined with $n_{0} \leftrightarrow n_{\infty}$ ), is tabulated for each $N$ from 0 to 24 in table 2. Note that in cases where the base is toric and could have either $N=1$ or $N=2$ (this can occur when there is a curve of selfintersection 0 that can either appear as a fiber or as one of $\left.D_{0}, D_{\infty}\right)$ we have counted the base as having the minimal value, $N=1$. We find a total of 126,469 smooth $\mathbb{C}^{*}$-bases that are acceptable for 6D F-theory compactifications. There are an additional 35,935 bases that come from $\mathbb{C}^{*}$-bases with $-9,-10$, and -11 curves in the fiber chains, for a total of 162,404 allowed bases. We use this larger set for the statistical analyses in the remainder of this paper. The largest fraction (nearly $38 \%$ ) of these bases come from the case with 3 


\begin{tabular}{|c|c|c|c|c|c|}
\hline \# Fibers $N$ & $\mathbb{C}^{*}$-bases & $\mathbb{C}^{*}+$ NSC bases & $\operatorname{Max} T$ & $\operatorname{Min} T$ & Peak $T$ \\
\hline $\mathbb{P}^{2}$ & 1 & 1 & 0 & 0 & 0 \\
\hline 0 & 10 & 10 & 1 & 1 & 1 \\
\hline 1 & 9,383 & 14,183 & 193 & 2 & 18 \\
\hline 2 & 25,474 & 28,733 & 182 & 3 & 21 \\
\hline 3 & 44,930 & 61,329 & 171 & 4 & 21 \\
\hline 4 & 20,980 & 27,134 & 160 & 5 & 21 \\
\hline 5 & 11,027 & 13,811 & 149 & 6 & 21 \\
\hline 6 & 6,137 & 7,462 & 138 & 7 & 25 \\
\hline 7 & 3,485 & 4,133 & 127 & 8 & 25 \\
\hline 8 & 2,034 & 2,356 & 116 & 9 & 25 \\
\hline 9 & 1,190 & 1,329 & 105 & 10 & 25 \\
\hline 10 & 709 & 768 & 94 & 11 & 25 \\
\hline 11 & 423 & 449 & 83 & 12 & 25 \\
\hline 12 & 262 & 273 & 72 & 13 & 25 \\
\hline 13 & 159 & 164 & 61 & 14 & 25 \\
\hline 14 & 101 & 104 & 61 & 15 & 25 \\
\hline 15 & 62 & 63 & 39 & 16 & 25 \\
\hline 16 & 40 & 40 & 30 & 17 & 25 \\
\hline 17 & 24 & 24 & 27 & 18 & 25 \\
\hline 18 & 16 & 16 & 26 & 19 & 25 \\
\hline 19 & 9 & 9 & 25 & 20 & 25 \\
\hline 20 & 6 & 6 & 25 & 21 & 25 \\
\hline 21 & 3 & 3 & 25 & 25 & 25 \\
\hline 22 & 2 & 2 & 25 & 25 & 25 \\
\hline 23 & 1 & 1 & 25 & 25 & 25 \\
\hline 24 & 1 & 1 & 25 & 25 & 25 \\
\hline total & 126,469 & 162,404 & 193 & 0 & 25 \\
\hline
\end{tabular}

Table 2. The number of distinct $\mathbb{C}^{*}$-bases, with two divisors $D_{0}$ and $D_{\infty}$ associated with sections $\Sigma_{ \pm}$connected by $N$ chains of curves of negative self-intersection. Cases $N=0,1,2$ correspond to toric bases. $P^{2}$ is listed separately. Toric bases with a single 0 -curve that can either be a section $(N=2)$ or a fiber $(N=1)$ are listed as $N=1$ bases. NSC refers to bases that are not strictly $\mathbb{C}^{*}$-surfaces in that they are described by $\mathbb{C}^{*}$-surfaces with $-9,-10$, or -11 curves in the fiber chains whose blow-up takes the base outside the set of $\mathbb{C}^{*}$-surfaces. The bases described using toric geometry in [12] all appear in this table, though some are not strictly toric and appear in column 3 and/or in rows with $N>2$ due to additional fibers produced when blowing up $-9,-10,-11$ curves. Peak $T$ refers to the value of $T$ that occurs for the greatest number of bases at each $N$. Both NSC and strictly $\mathbb{C}^{*}$-surfaces were used to determine the maximum, minimum, and peak $T$ values.

non-trivial chains, and the number of allowed bases decreases as the number of non-trivial chains increases above 3 .

As mentioned above, the tabulation of bases given in table 2 differs from that of [12] 
in the way that toric bases with $-9,-10$, and -11 curves are treated. In [12], 61,539 bases were identified based on toric structures that in some cases included $-9,-10$, or -11 curves. These bases include the 34,868 strictly toric bases listed for $\mathbb{P}^{2}$ and $N=0,1$ and 2 in column 2 of table 2, another 8,059 bases that arise from toric bases with $-9,-10$, or -11 curves on the fiber chains, included in column 3 of the table, and another 18,612 bases that are included in rows $N>2$ and correspond to $\mathbb{C}^{*}$-bases (with or without $-9,-10$, or -11 curves on the fiber chains). Bases in the last category arise from toric bases with $-9,-10,-11$ curves on the sections $D_{0}, D_{\infty}$ that give extra nontrivial fibers when the necessary points are blown up. To summarize, the analysis here includes all the bases constructed in [12], but discriminates more precisely based on the detailed structure of these bases.

The parameter $T$ (number of tensor multiplets) serves as the most significant distinguishing characteristic of $6 \mathrm{D}$ supergravity theories, and is related to the simplest topological feature $h^{1,1}(B)$ of the base surface $B$. Since each nontrivial fiber involves at least one blowup, and $T=1$ for all $\mathbb{F}_{m}, T \geq N+1$. The value of $T=h^{1,1}(B)-1$ for the $\mathbb{C}^{*}$-bases with $N>2$ ranges from $T=4$ to $T=171$. The largest $T$ for any known base is $T=193$; this is an NSC base with only one nontrivial fiber $(N=1)$. Heuristic arguments were given in [12] that no other base can have $T>193$; as discussed in the next section this conclusion is supported by the results of this paper. Note that for $N$ from 1 to 13, the maximum value of $T$ drops by precisely 11 for each increment in $N$. This can be understood from the appearance of specific maximal sequences containing -12 curves, as discussed in section 3.3.

The number of different bases that appear at each $T$ is plotted in figure 5 . The number of bases peaks at $T=25$. The distribution of bases also peaks at $T=25$ for every specific $N>5$, out to $N=21,22,23$ and 24 , where all possible $\mathbb{C}^{*}$-bases have $T=25$. One feature of many of the bases with $T=25$ is that they contain precisely two $(-12)$ clusters giving $\mathfrak{e}_{8}$ gauge summands, and no other non-Higgsable clusters. The primary difference between these bases is that they have different numbers of nontrivial chains and contain different combinations of intersecting -2 curves. As mentioned above, clusters of -2 curves without curves of lower self-intersection generally arise at special points in moduli spaces of bases without such clusters. Indeed, many of the $T=25$ bases with gauge algebra $\mathfrak{e}_{8} \oplus \mathfrak{e}_{8}$ are limits of the same complex geometry. We discuss this issue further in section 4.4, in the context of the full Calabi-Yau threefold geometry of the elliptic fibration over $B$. In a similar fashion, many bases have $T=21$ and contain one $(-12)$ cluster and a single $(-8)$ cluster associated with a $\mathfrak{e}_{7}$ algebra summand.

The number of $\mathbb{C}^{*}$-bases drops off rapidly between about $T=30$ and $T=60$. Similar behavior for the toric subset of the bases was noted in [12]. In addition to the primary peak at $T=25$, there are smaller peaks in the distribution of bases starting at $T=50$ and appearing at intervals of eleven, so that they are visible at $T=61,72,83,94$, etc. This feature is also visible when we consider the $N$-chain cases separately for $3 \leq N \leq 14$ (see figure 6). Again, the increment by 11 is related to $\mathfrak{e}_{8}$ chain sequences, though the geometry seems less restricted as $T$ increases.

It may seem surprising that the number of possible bases drops off so rapidly with 


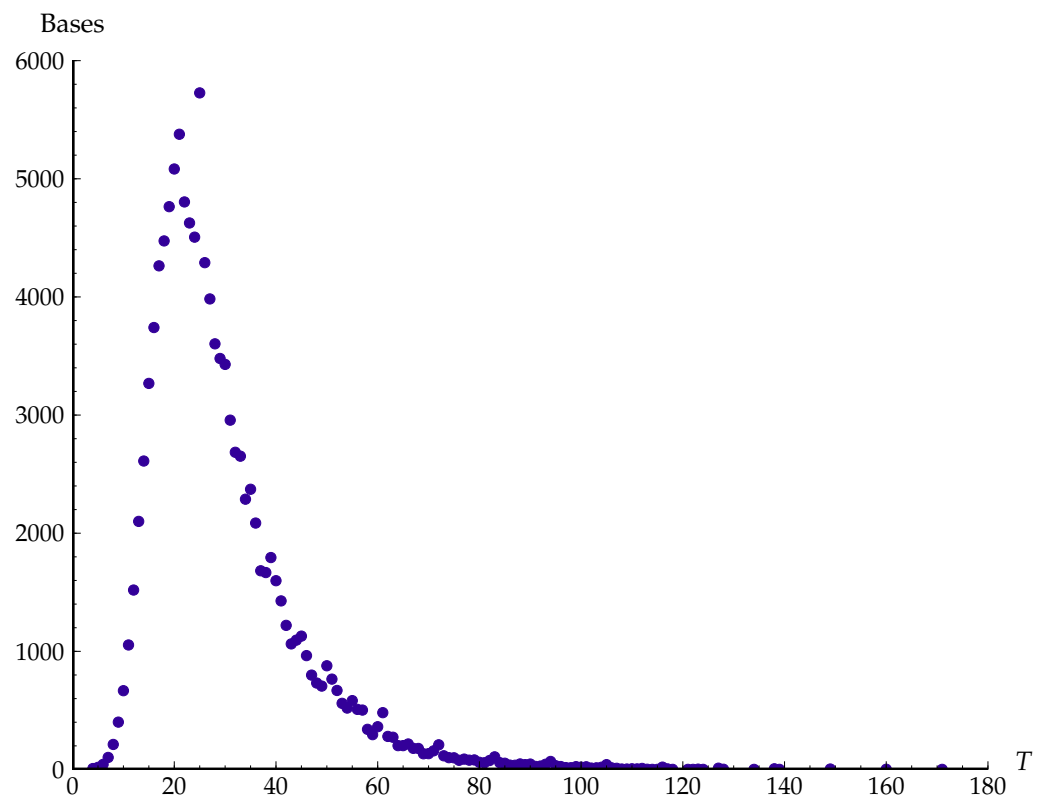

Figure 5. Number of distinct $\mathbb{C}^{*}$-bases as a function of the number of tensor multiplets $T$.

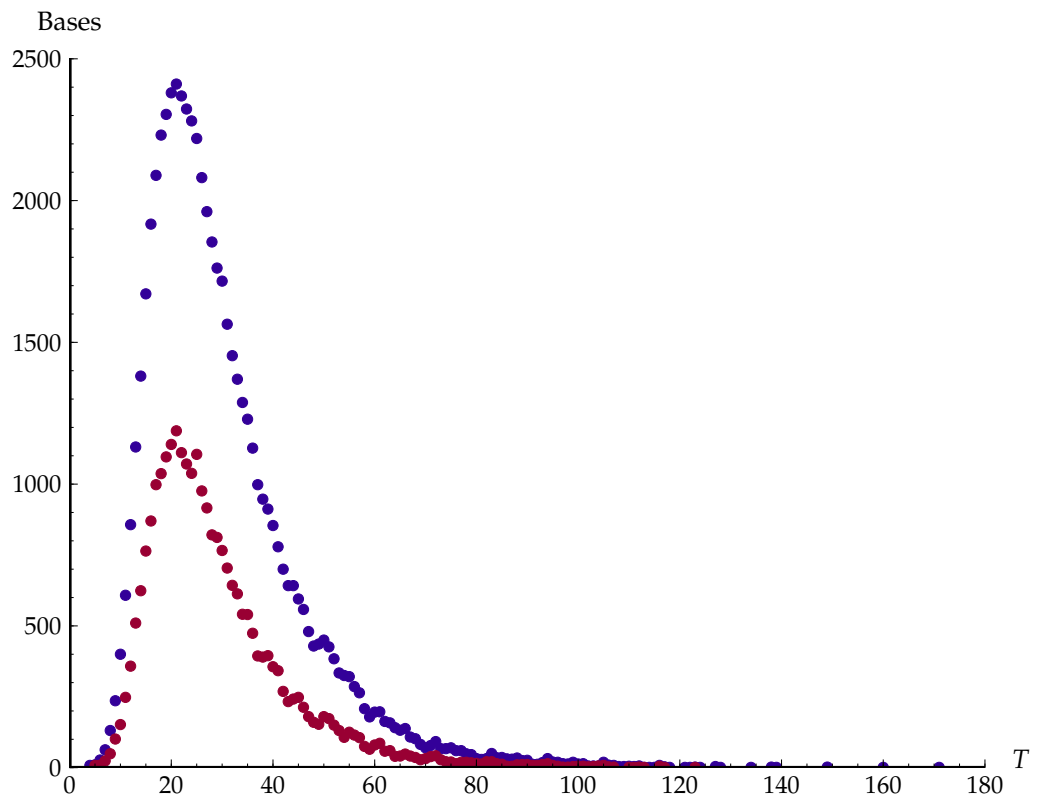

Figure 6. Number of distinct $\mathbb{C}^{*}$-bases with $N=3$ Fibers (upper blue data) and $N=4$ Fibers (lower purple data) for different numbers $T$ of tensor multiplets. 


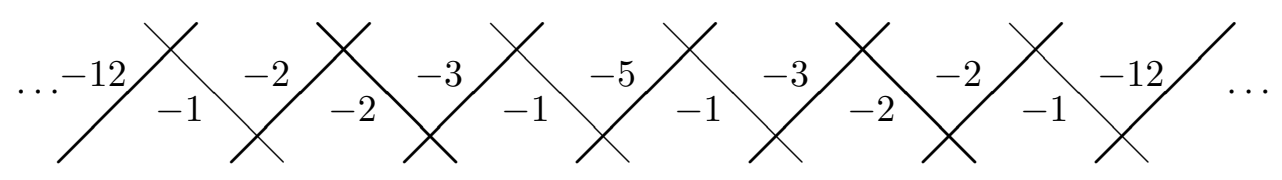

Figure 7. Bases having large values of $h^{11}(B)=T+1$ have an intersection structure dominated by multiple repetitions of a characteristic sequence of intersecting divisors associated with nonHiggsable gauge algebra $\mathfrak{e}_{8} \oplus \mathfrak{f}_{4} \oplus 2\left(\mathfrak{g}_{2} \oplus \mathfrak{s u}(2)\right)$.

$N$. Naively, one might imagine a set of $K$ relatively short chains that could be combined arbitrarily in roughly $K^{N} / N$ ! ways, which could grow rapidly with increasing $N$ for even modest values of $K$. The fact that the number of bases decreases rapidly for increasing $N$ indicates that in fact, not many chains can be combined arbitrarily. These constraints come in part from the intersection rules that drastically reduce the possibilities of which chains can simultaneously intersect one of the sections, and in part from the increased number of blow-ups at points on the sections that are needed to build complicated chains. The relatively controlled dependence on $N$ of the number of models suggests that going beyond the class of $\mathbb{C}^{*}$-surfaces to completely generic base surfaces may also give a reasonably controlled number of possible bases.

\subsection{Gauge groups and chain structure}

We have investigated a number of aspects of the class of $\mathbb{C}^{*}$-bases that we have identified. One of the main conclusions of this investigation is that the structure of the $\mathbb{C}^{*}$-bases with large $T$ is very similar to that of toric bases with large $T$. For toric bases with large $T$, the intersection structure of divisors with negative self-intersection is largely based on long chains dominated by sequences of maximal units with gauge algebra $\mathfrak{e}_{8} \oplus \mathfrak{f}_{4} \oplus 2\left(\mathfrak{g}_{2} \oplus \mathfrak{s u}(2)\right)$ (See figure 7). The same is true of $\mathbb{C}^{*}$-bases. The number of gauge algebra summands of these types are plotted in figure 8 against $T$, and grow linearly in a fashion very similar to that in the toric case. The linear sequences of 12 curves giving the gauge algebra contributions $\mathfrak{e}_{8} \oplus \mathfrak{f}_{4} \oplus 2\left(\mathfrak{g}_{2} \oplus \mathfrak{s u}(2)\right)$ were described in [10]; we refer to these as "ép 8 units" for simplicity, as they appear frequently in the intersection structure of bases with large $T$. These units are maximal in the sense that they can not be blown up in any way consistent with the existence of an elliptic fibration.

A detailed analysis of the configurations at large $T$ makes this correspondence clear. As discussed in $[10,12]$, the base with $T=193$ is a (NSC) base with $N=1$, where the single fiber chain is essentially $16 \mathfrak{e}_{8}$ units ending in -12 curves at $D_{0}, D_{\infty}$; the two -12 curves one $\mathfrak{e}_{8}$ unit away from the ends of the fiber are actually -11 curves in the toric base, which is what makes this base not strictly toric or $\mathbb{C}^{*}$ (and therefore places it in the third column of table 2). The $N=2$ base with $T=182$ is identical except that the long fiber 


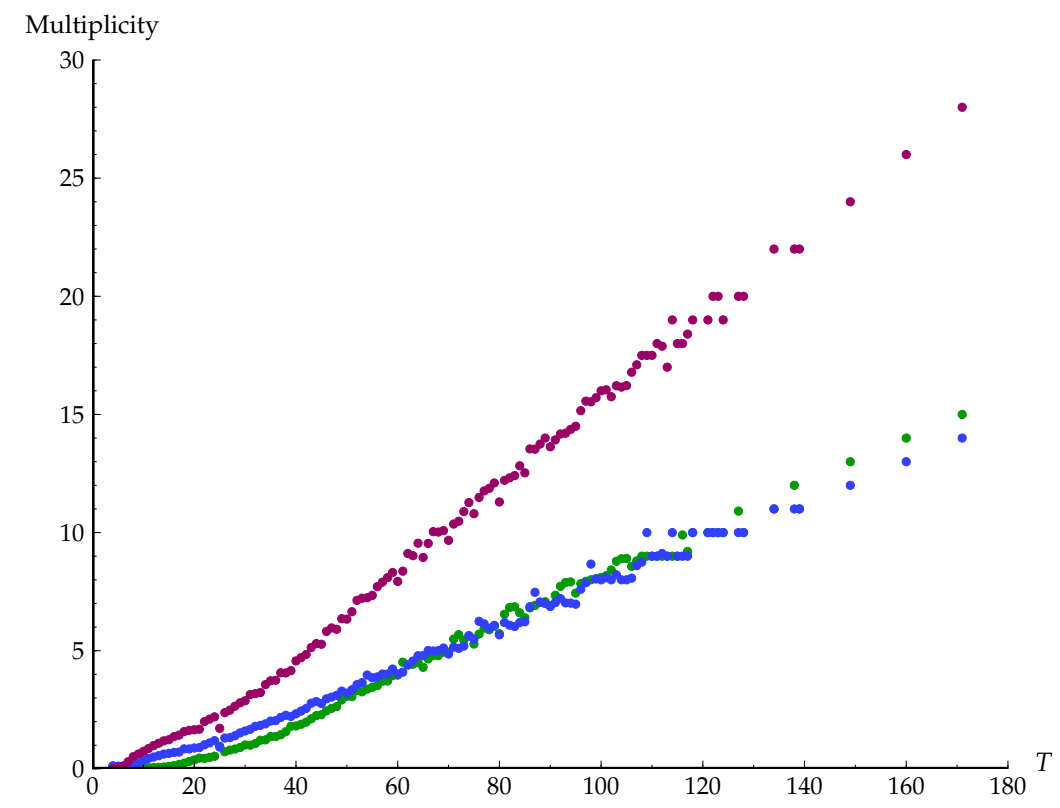

Figure 8. Average number of gauge algebra summands as a function of $T$ for summands (ordered from top at $T=171) \mathfrak{g}_{2} \oplus \mathfrak{s u}(2), \mathfrak{e}_{8}$, and $\mathfrak{f}_{4}$.

chain contains only $15 \mathfrak{e}_{8}$ units (again with -11 curves one $\mathfrak{e}_{8}$ unit away from each end), and the second chain is a simple $(-1,-1)$ chain arising from a single blow-up on one of the sections. The unique $N=3 \mathbb{C}^{*}$-base with $T=171$ is again similar, with $n_{0}=n_{\infty}=-12$, two $(-1,-1)$ chains, and the longer chain being a sequence of $14 \mathfrak{e}_{8}$ units, again with two -11 curves in the $\mathbb{C}^{*}$-base. Other bases with large $T$ are dominated in a similar fashion by one or two very long chains, with the other chains generally being of type $(-1,-1)$ or other very short chains. For example, there is only one base with $N=3$ that has two fiber chains of length $>50$ (see figure 9 ). For this base there are two long chains of length 53 that combine to form a loop of $9 e_{8}$ chains, with a -12 curve and the opposite -5 curve for $D_{0}$ and $D_{\infty}$ (and the usual pair of -11 curves near the end on each side), and the third fiber chain is of the form $(-1,-1)$. Over all $N=3$ bases, when the shortest fiber chain has length $>3$ then the next shortest is of length 23 or less.

This analysis of large $T \mathbb{C}^{*}$-bases strengthens the conclusion argued heuristically in [12] that no base can have $T>193$. In particular, the $\mathbb{C}^{*}$-bases allow two new types of topological structure to the intersection diagram: loops and branches. Neither of these types of structure allows for new constructions that qualitatively change the nature of the bases at large $T$. In general, bases with large $T$ have a long linear sequence of $\mathfrak{e}_{8}$ chains, with additional loops rapidly decreasing the maximum value of $T$. There are no new features associated with branches that suggest any mechanism for constructing bases with large $T$ and more complicated intersection structures. In fact, the known base with the largest $T$ corresponds to simply a single long $\mathfrak{e}_{8}$ chain, with no branching or loops at all. The only way to modify this structure topologically is to add branchings and loops, and the results that we have found here show that adding up to two branchings and an 


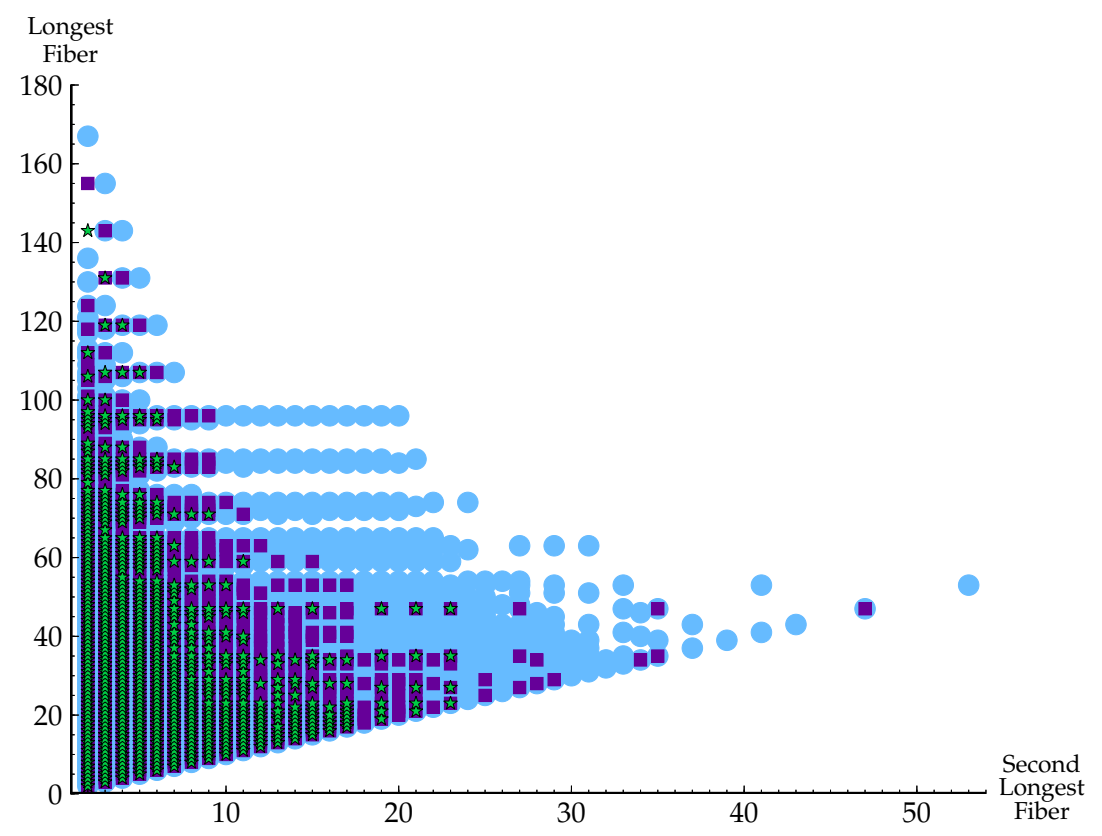

Figure 9. Comparison of the lengths of the longest and second longest chains associated with distinct fibers for bases with $N=3$ (blue circles), $N=4$ (purple squares), and $N=5$ (green stars).

arbitrary number of loops does not give any way of increasing $T$; rather, as the topological complexity increases the upper bound on possible $T$ 's decreases. Thus, while we still do not have a proof that $T=193$ is the maximum possible for a smooth base $B$, the absence of new structure in the $\mathbb{C}^{*}$-base models seems to make it unlikely that generic bases with even more complicated branching and looping structures could have larger values of $T$, even without the $\mathbb{C}^{*}$ restriction.

\section{Calabi-Yau geometry}

Over each F-theory base it is possible to construct an elliptically fibered Calabi-Yau geometry. Resolving singularities associated with the nonabelian groups living on the discriminant locus gives a smooth Calabi-Yau threefold. While tuning the moduli over any base can increase the degree of vanishing of the discriminant locus and hence enhance the nonabelian gauge group, which corresponds to a change in the associated smooth Calabi-Yau threefold, we focus here primarily on the simplest threefold over each base, corresponding to the maximally Higgsed 6D supergravity model.

Each Calabi-Yau threefold $X$ has topological invariants given by the Hodge numbers $h^{1,1}(X), h^{2,1}(X)$. A large class of Calabi-Yau threefolds that are realized as hypersurfaces in toric varieties were identified by Kreuzer and Skarke [9], who produced a comprehensive database of 474 million Calabi-Yau constructions of this type. These examples include 


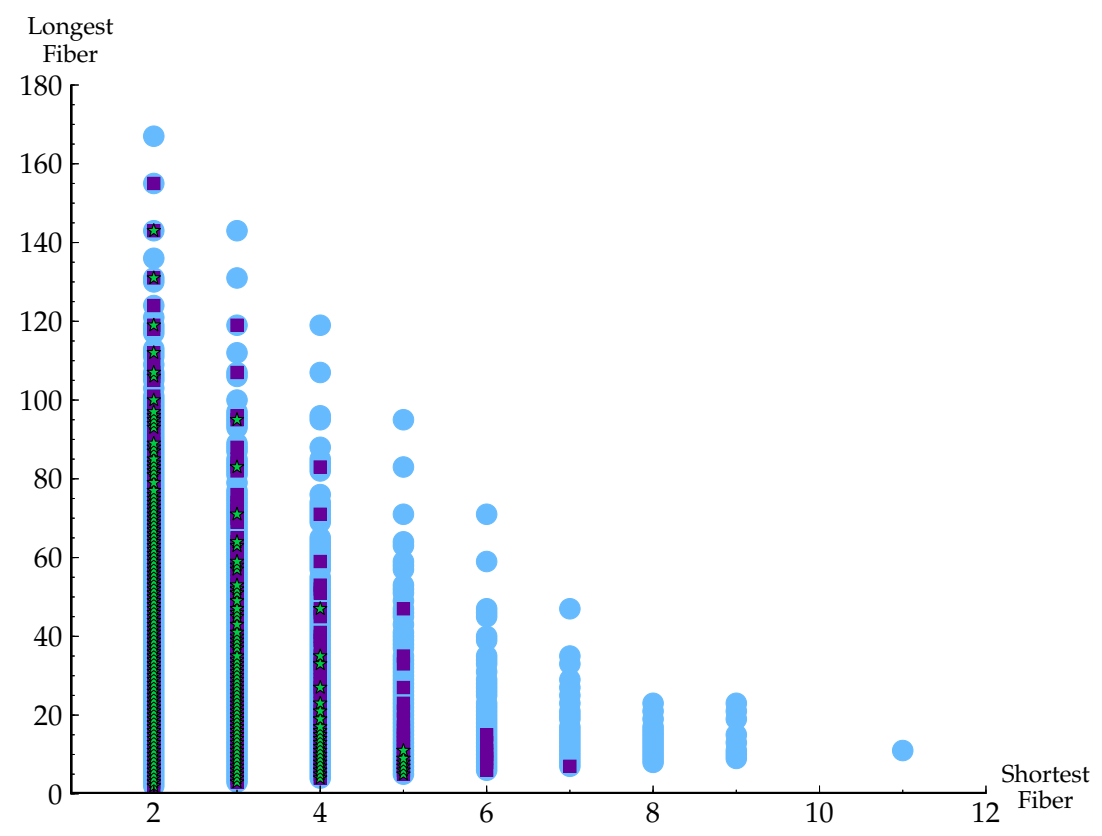

Figure 10. Comparison of the lengths of the longest and shortest chains associated with distinct fibers for bases with $N=3$ (blue circles), $N=4$ (purple squares), and $N=5$ (green stars).

threefolds with 30,108 distinct pairs of Hodge numbers. The Hodge numbers associated with allowed toric bases (including some NSC bases) were computed in [13], and it was shown that the distinctive upper boundary of the "shield region" spanned by the Kreuzer and Skarke Hodge data is associated with a trajectory of blow-ups of the Hirzebruch surface $\mathbb{F}_{12}$. In particular, it was proven that the maximum possible value of $h^{2,1}(X)$ for any elliptically-fibered Calabi-Yau threefold (with section) is given by

$$
h^{2,1}(X) \leq 491
$$

independent of whether the base is toric or not. In this section we carry out an analysis of the Hodge numbers for the more general class of $\mathbb{C}^{*}$-bases, allowing us to identify some new Calabi-Yau threefolds with interesting features.

\subsection{Calabi-Yau geometry of elliptic fibrations over $\mathbb{C}^{*}$-bases}

The Hodge numbers of the smooth Calabi-Yau threefold $X$ associated with a generic elliptic fibration over the base $B$ are related to the intersection structure of the base and to the gauge group and matter content of the associated $6 \mathrm{D}$ supergravity theory.

The Hodge number $h^{1,1}(X)$ is related to the structure of the base by the Shioda-TateWazir formula [28], which in the language of the $6 \mathrm{D}$ supergravity theory states $[4,5]$

$$
h^{1,1}(X)=h^{1,1}(B)+\operatorname{rank}(G)+1=T+2+\operatorname{rank}\left(G_{\text {nonabelian }}\right)+V_{\text {abelian }},
$$


where $G$ is the full (abelian + nonabelian) gauge group of the $6 \mathrm{D}$ theory. While the nonabelian group is determined as described above from the singularity structure of the discriminant locus, the rank of the abelian group corresponds to the rank of the MordellWeil group of sections of the fibration. In general, the rank of the Mordell-Weil group is difficult to compute mathematically (see, e.g., [29]). The Mordell-Weil group is a global feature of the elliptic fibration, corresponding in the physics context to the number of $\mathrm{U}(1)$ factors in the $6 \mathrm{D}$ supergravity theory; the global aspect of this structure is what makes computation of the group a particularly challenging problem. Recent progress on understanding abelian factors in general F-theory constructions was made in [30-44].

The Hodge number $h^{2,1}(X)$ gives the number of complex structure moduli of the threefold $X$. This set of moduli represents all but one of the neutral scalar fields in the $6 \mathrm{D}$ gravity theory - the remaining scalar field is associated with the overall Kähler modulus on the base $B$.

$$
h^{2,1}(X)=H_{\text {neutral }}-1 .
$$

The number of neutral hypermultiplets in the theory on a $\mathbb{C}^{*}$-base $B$ can be computed from the intersection structure on $B$ by analyzing the generic Weierstrass form over that base, as we describe below in section 4.2. The total number of scalar fields is also related through the $6 \mathrm{D}$ gravitational anomaly cancellation condition to the number of vector multiplets $V$ in the theory $[45,46]$

$$
H_{\text {neutral }}+H_{\text {charged }}-V=273-29 T .
$$

Since the number of charged hypermultiplets can be computed by adding the contributions from table 1 for each non-Higgsable cluster in $B$, and the numbers of neutral hypermultiplets, tensors, and nonabelian vectors are also computable from the intersection data on $B$, this gives us a way of computing the total number of abelian vector multiplets $V_{\text {abelian. }}$ Using this in (4.2), we can thus compute both Hodge numbers $h^{1,1}(X), h^{2,1}(X)$ in a systematic way for any $\mathbb{C}^{*}$-base.

\subsection{Counting neutral hypermultiplets}

Computing the number of neutral hypermultiplets over a given base $B$ can be done in a systematic fashion by following the sequence of blow-ups needed to reach $B$ starting from a Hirzebruch surface $\mathbb{F}_{m}$. In the case where $B$ is a toric surface, this analysis is particularly simple and was described in [12]. For a toric base, the number of neutral hypers is related to the number of free parameters $W$ in the Weierstrass model for $B$ through

$$
H_{\text {neutral }}=W-w_{\text {aut }}+N_{-2} \quad(\text { toric }),
$$

where $w_{\text {aut }}$ is the dimension of the automorphism group of $B$, and $N_{-2}$ is the number of curves of self-intersection -2 in $B$ that do not live in clusters carrying a gauge group. Basically, automorphisms of $B$ correspond to Weierstrass monomials that do not represent physical degrees of freedom, while -2 curves represent moduli that have been tuned to a special point, as discussed above. As shown in [12], each curve of self-intersection $k \geq 0$ in the toric fan contributes $k+1$ to the dimension of the automorphism group, with two additional universal automorphisms corresponding to the toric structure. For toric surfaces, 
the number of Weierstrass monomials in $f$ is simply given by the set of points $m$ in the lattice dual to the toric fan that satisfy $\langle m, v\rangle \geq-4$ for all $v$ in the set of rays generating the fan, and a similar condition with $\langle m, v\rangle \geq-6$ for monomials in $g$. As discussed in [12], there is a simple geometric picture of this in the toric context.

More generally, we can start on $\mathbb{F}_{m}$ with a given number of monomials $W$ in the Weierstrass degrees of freedom of $f, g$ and explicitly tune the moduli in $f, g$ to blow up the base at the desired set of points to realize any base $B$. We can then compute the number of remaining Weierstrass moduli and apply (4.5) (with one slight modification in the counting of $N_{-2}$ as discussed further below). This procedure can be carried out in a clear fashion when the base $B$ is $\mathbb{C}^{*}$. The simplification in the $\mathbb{C}^{*}$ case comes from the fact that we can treat each chain arising from a blown-up fiber on $\mathbb{F}_{m}$ in a parallel fashion to the toric case. In the toric case we are blowing up points on only two fibers, which we can take to be at the points $z=0, \infty$ in a local toric coordinate chart $(z, w) \in \mathbb{C} \times \mathbb{C}$ as discussed in section 2.3. Consider the constraints on Weierstrass monomials coming from blow-ups along the fiber at $z=0$. Each such constraint corresponds to imposing a vanishing condition on a set of monomial coefficients in an expansion $f=\sum_{n, m} c_{n, m} z^{n} w^{m}$, and similarly for $g$. The set of constraints imposed depends upon the sequence of blow-ups in the fiber, and has a convenient geometric description in the language of the toric fan and dual space. For a $\mathbb{C}^{*}$-base with $N$ chains located at $z=z_{1}, \ldots, z_{N}$, we simply impose the appropriate set of constraints on the monomial coefficients in an expansion $f=\sum_{n, m} c_{n, m}^{(i)}\left(z-z_{i}\right)^{n} w^{m}$ for the $i$ th chain. This gives a set of linear conditions on the coefficients in $f, g$. The dimension of the space of independent solutions to these linear constraints can then be used to compute the number of independent Weierstrass monomials $W$, in an analogous fashion to (4.5). As in the toric case, when one of the sections $D_{0}, D_{\infty}$ has self-intersection $k \geq 0$ it contributes $k+1$ to the dimension of the automorphism group. For a $\mathbb{C}^{*}$-base with $N=3$ or more blown up fibers, the first two fibers can be fixed at $z=0, \infty$, using up two automorphisms originally associated with the fibers of self-intersection 0 in the toric base. The blowing up of the third fiber corresponds to fixing another point in $\mathbb{C}^{*}$, which can for example be chosen to be $z=1$, fixing one of the remaining automorphisms and leaving only one of the universal two present in all toric bases. For each of the $N-3$ fibers beyond the third, blowing up an additional point involves choosing a value $z_{i}$ which is itself a Weierstrass parameter though it does not correspond to a monomial. Thus, the formula (4.5) must be augmented by $N-3$ when more than 3 fibers are blown up. The complete formula for the number of neutral hypermultiplets is given at the end of this section, in (4.12).

It may be helpful to illustrate this method with an example. Consider first the toric base $B$ with the following parameters

$$
\begin{aligned}
N=2, & n_{0}=-2, n_{\infty}=-1 \\
\text { chain } 1: & (-1,-3,-1,-2) \\
\text { chain 2: } & (-1,-1)
\end{aligned}
$$

This base has one $(-3)$ cluster, so the nonabelian gauge algebra is $\mathfrak{s u}(3)$. This base can be described as $\mathbb{F}_{0}$ with four points blown up, three on the fiber $z=0$ and one on the fiber at 

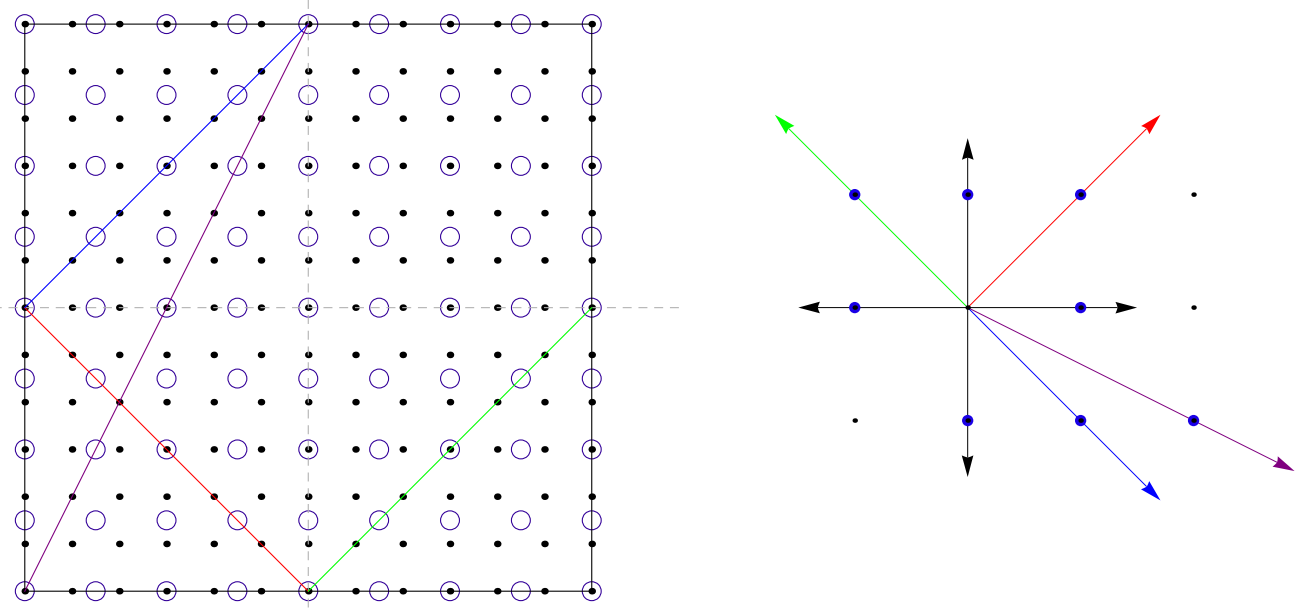

Figure 11. An example of counting monomials in a toric base $B$. The base $\mathbb{F}_{0}$ has 250 Weierstrass coefficients: $9 \times 9=81$ in $f$ (solid dots) and $13 \times 13$ in $g$ (round circles) in the dual lattice. These are all of the dots and circles in the diagram on the left. Each blow-up corresponds to adding a vector to the fan (above right), which removes some monomials from $f$ and $g$. For example, blowing up the point corresponding to the red ray in the diagram on the right removes all monomials below and to the left of the red line in the left-hand diagram, blowing up on the blue ray removes all points above and to the left of the blue line, etc.. The base $B$ has 136 monomials, 44 in $f$ and 92 in $g$, corresponding to the dots and circles in the left-hand diagram that lie above the red and green lines and to the right of the purple line.

$z=\infty$. A graphic description of the toric fan and monomials in the dual lattice is given in figure 11 .

The monomials in this toric model can be computed using the methods of [12]. The monomials in $f$ in the Weierstrass model on $\mathbb{F}_{0}$ are of the form $c_{n, m} z^{n} w^{m}, 0 \leq n, m \leq 8$, and similarly for $g=\sum_{n, m=0}^{12} d_{n, m} z^{n} w^{m}$. Blowing up the points on $\mathbb{F}_{0}$ imposes conditions on the coefficients $c, d$. For example, blowing up the point $z=w=0$ (corresponding to adding the ray in red in the diagram on the right-hand side of figure 11) imposes the conditions that $c_{n, m}=0$ for all $n+m<4$ and $d_{n, m}=0$ for $n+m<6$. This removes 31 Weierstrass moduli (and reduces the automorphism group by 2 by turning two 0 -curves into -1-curves), giving a change in the number of neutral hypermultiplets $\Delta H_{\text {neutral }}=-29$, matching (4.4) with $\Delta T=1$. This corresponds to removing all the monomials on the lower-left corner of the left-hand diagram in figure 11. The analogous constraints imposed by blowing up the other points on the fiber $z=0$ as well as the point $z=\infty, w=0$ are depicted in an analogous fashion in the figure. Together, these constraints reduce the number of Weierstrass monomials to $W=136$. This matches with (4.5) and (4.4), with $N_{-2}=w_{\text {aut }}=2$ and $V=8$ vector multiplets from the $\mathfrak{s u}(3)$ factor in the gauge algebra. Thus, we have determined the Hodge numbers of the resolved generic elliptic fibration over $B$

$$
h^{1,1}(X)=9, \quad h^{2,1}(X)=135 .
$$

Now, consider blowing up a point on a third fiber at $z=1$ to form the $\mathbb{C}^{*}$-base $B^{\prime}$ shown in figure 12. Blowing up at the point $(z, w)=(1,0)$ imposes the condition that $f$ and $g$ must vanish to degrees 4 and 6 in $(z-1)$ and $w$. Consider for example $f$. From the geometry depicted in figure 11 it is clear that the only undetermined coefficients of $f$ at 


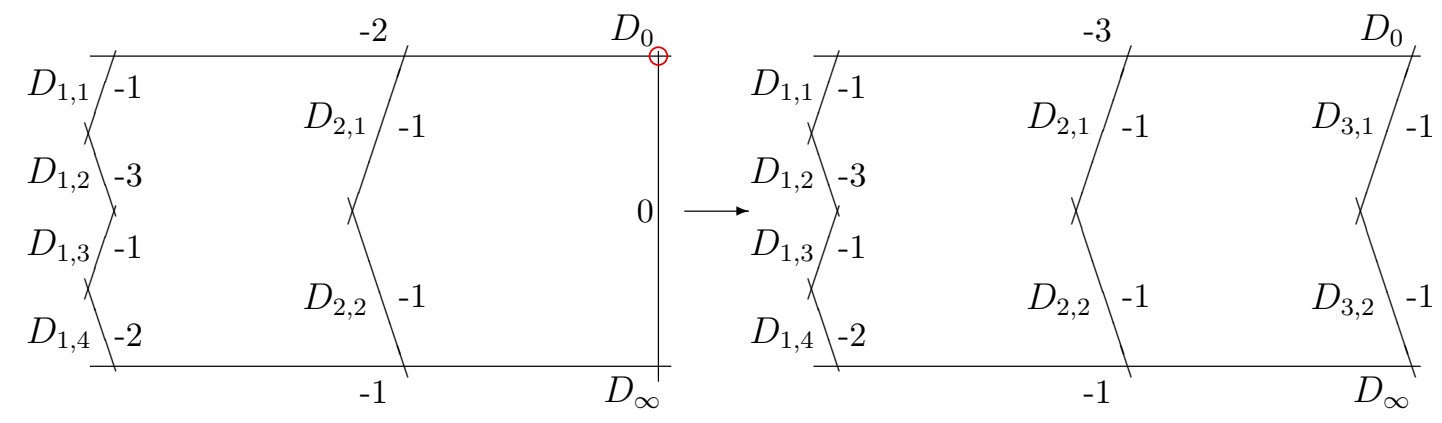

Figure 12. An example of a $\mathbb{C}^{*}$-surface $B^{\prime}$ given by blowing up the toric surface $B$ from figure 11 at a point.

leading orders in $w$ are

$$
f=c_{4,0} z^{4}+c_{3,1} z^{3} w+c_{4,1} z^{4} w+c_{5,1} z^{5} w+\sum_{n=2}^{6} c_{n, 2} z^{n} w^{2}+\cdots
$$

The condition that this vanishes to degree 4 in $(z-1), w$ forces all coefficients $c_{n, 0}$ and $c_{n, 1}$ to vanish, imposes two constraints at $m=2$, and one constraint at $m=3$, totaling 7 new constraints. Similarly, coefficients of $g$ experience 14 further constraints, for a total of 21 constraints. This reduces the number of Weierstrass monomials to $W=115$. The equations (4.5) and (4.4) still apply, where now $w_{\text {aut }}=1$ since one of the toric automorphisms is broken by the reduction to $\mathbb{C}^{*}$ structure, and $N_{-2}=1$ because the blow-up at $z=1, w=0$ changes $n_{0}$ from -2 to -3 . With one new -3 curve the number of vector multiplets becomes 16 , and the resolved generic elliptically fibered Calabi-Yau $X^{\prime}$ over $B^{\prime}$ has Hodge numbers

$$
h^{1,1}\left(X^{\prime}\right)=12, \quad h^{2,1}\left(X^{\prime}\right)=114 .
$$

In this way we can determine the Hodge numbers of the Calabi-Yau threefolds associated with generic elliptic fibrations over all $\mathbb{C}^{*}$-bases. When the additional fibers added are more complicated, the conditions on $f, g$ at $z=z_{i}$ can be determined by simply translating the conditions at $z=0$ from the toric picture.

The one remaining subtlety in this general picture is that for general $\mathbb{C}^{*}$-bases some combinations of -2 curves must be treated specially. ${ }^{4}$ In particular, for certain configurations of intersecting -2 curves associated with Kodaira-type surface singularities there is a linear combination that describes a degenerate genus one curve [20]. In these cases, the extra deformation directions associated with the -2 curves are not independent and the contribution from $N_{-2}$ to (4.5) is reduced by 1 . The -2 curve configurations of these types that appear in $\mathbb{C}^{*}$-bases are shown in figure 13 . These -2 curve configurations can be identified as those where an integral linear combination of the -2 curves is a divisor with vanishing self-intersection. For this to occur, the weighting of any -2 curve $C_{i}$ must be $1 / 2$ the total of the weightings of the -2 curves that intersect $C_{i}$. Some simple combinatorics

\footnotetext{
${ }^{4}$ Thanks to David Morrison for discussions on this point
} 


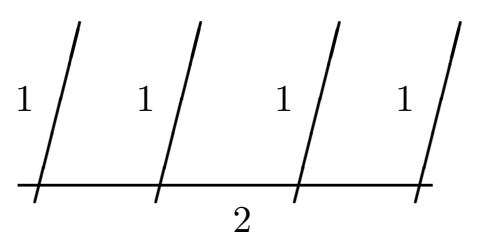

(a) $I_{0}^{*}\left(\hat{D}_{4}\right)$

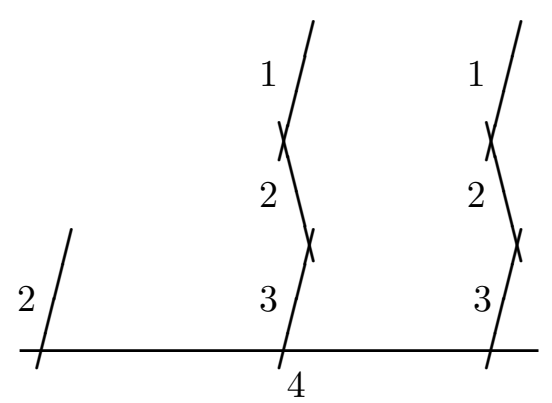

(c) $I I I^{*}\left(\hat{E}_{7}\right)$

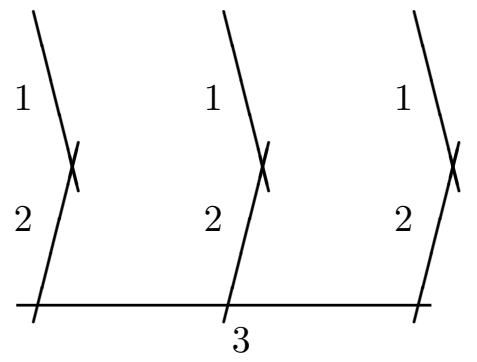

(b) $I V^{*}\left(\hat{E}_{6}\right)$

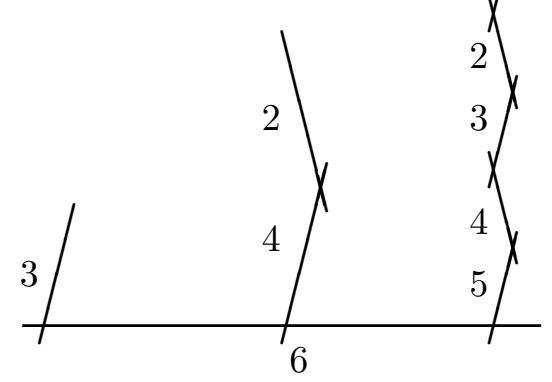

(d) $I I^{*}\left(\hat{E}_{8}\right)$

Figure 13. Configurations of -2 curves associated with Kodaira-type surface singularities associated with degenerate elliptic fibers. For these configurations, the number of fixed moduli associated with -2 curves is reduced by one. The numbers given are the weightings needed to give an elliptic curve with vanishing self-intersection. Labels correspond to Kodaira singularity type and associated Dynkin diagram.

shows that the configurations in figure 13 are the only possible geometries satisfying this condition that have a single -2 curve that intersects more than two others. ${ }^{5}$ Thus, for $\mathbb{C}^{*}$-bases that are not toric $(N \geq 3)$, the formula (4.5) is replaced by

$$
H_{\text {neutral }}=W-w_{\text {aut }}+(N-3)+N_{-2}-G_{1} \quad\left(\mathbb{C}^{*}\right),
$$

where $w_{\text {aut }}=1+\max \left(0,1+n_{0}, 1+n_{\infty}\right), N_{-2}$ is the number of -2 curves, and $G_{1}$ is the number of -2 configurations of the types shown in figure 13 .

\subsection{Distribution of Hodge numbers}

We have computed the Hodge numbers for the generic threefolds over all 162,404 $\mathbb{C}^{*}$-bases, including those not strictly $\mathbb{C}^{*}$-bases coming from blown up $\mathbb{C}^{*}$-bases with $-9,-10$, and -11 curves on the fiber chains. We find a total of 7,868 distinct pairs of Hodge numbers $h^{1,1}, h^{2,1}$, including 344 Hodge number combinations not found in [13] from the set of generalized toric bases. These Hodge numbers are plotted and compared to the KreuzerSkarke database [9] and the Hodge numbers of threefolds associated with toric bases in

\footnotetext{
${ }^{5}$ One other class of configurations satisfies this condition, the type $I_{b}^{*}$ singularity, with two -2 curves each intersecting three -2 curves and connected by a single chain of -2 curves, but this cannot appear in a $\mathbb{C}^{*}$-base since the chain associated with each fiber must contain at least one -1 curve, and in this case the chain connecting the two -2 curves with triple branching contains itself only -2 curves.
} 


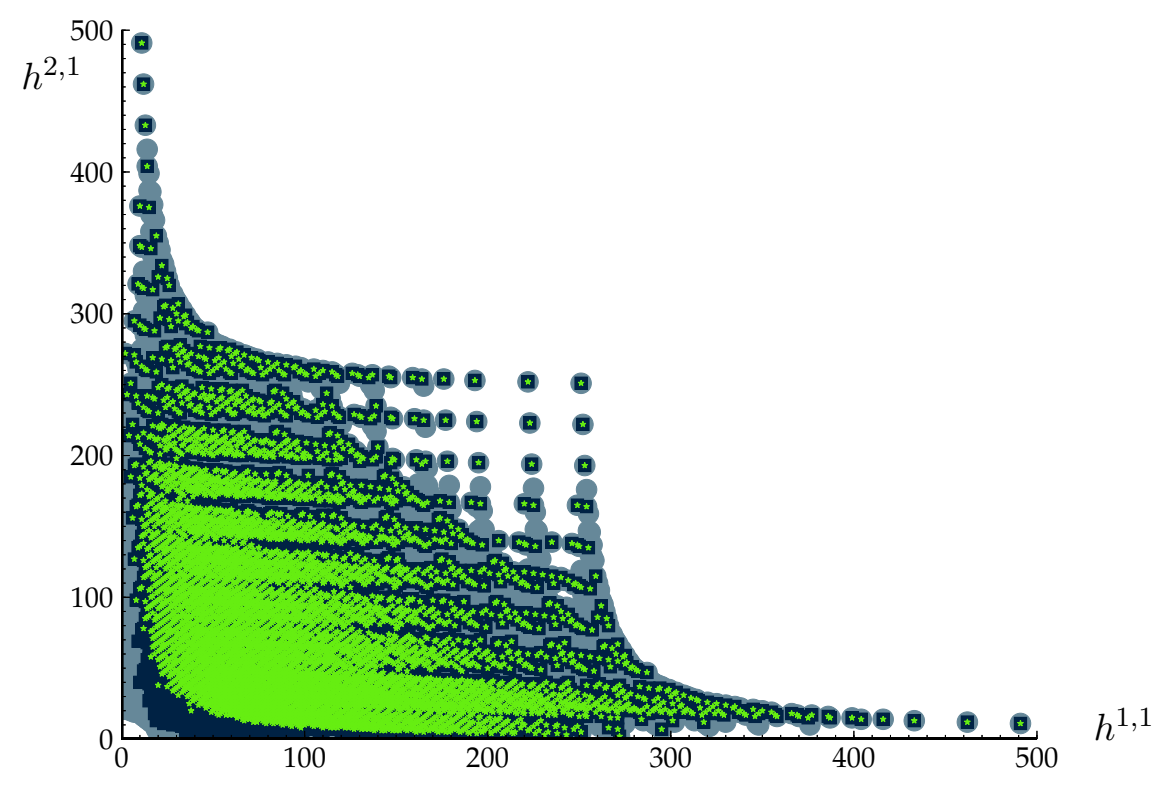

Figure 14. Plot of Hodge numbers comparing $\mathbb{C}^{*}$ (shown in dark blue, largest dots), KreuzerSkarke (slate blue, medium dots), and toric (green, smallest dots). Hodge numbers for $\mathbb{C}^{*}$-bases that are not also Hodge numbers for toric (or NS-toric) bases are generally those with small $h^{1,1}, h^{2,1}$ with a small number of exceptions having larger Hodge numbers, including 6 examples that are not found in the Kreuzer-Skarke database.

figure 14. The number of models as a function of the sum $h^{1,1}+h^{2,1}$ is plotted in figure 15 . Most of the new Hodge numbers that appear for $\mathbb{C}^{*}$-bases and not for toric bases are in the region of small Hodge numbers far from the boundary.

Note that just as for toric bases, as discussed in [12], the set of Calabi-Yau manifolds that can be constructed over any given $\mathbb{C}^{*}$-base $B$ can be very large. By tuning parameters in the Weierstrass model over any given base, so that $f$ and $g$ vanish on certain divisors to degrees less than 4,6 , theories with many different nonabelian gauge group factors can be constructed. Each such construction gives a different Calabi-Yau threefold after the singularities in the elliptic fibration are resolved. In this way, the number of Hodge numbers associated with Calabi-Yau threefolds fibered over any given $\mathbb{C}^{*}$-base can be quite large. Explicit examples of such tunings that give Hodge numbers near the boundary of the "shield" for threefolds over toric bases are described in [13, 47]. Similar constructions over $\mathbb{C}^{*}$-bases would give a vast range of different Calabi-Yau threefold constructions.

\subsection{Redundancies from -2 clusters}

One striking feature of the distribution of Hodge numbers is that there are certain Hodge number combinations that are realized by the threefolds associated with a large number of distinct $\mathbb{C}^{*}$-bases. As the most extreme example, there are 1,861 different $\mathbb{C}^{*}$-bases with Hodge numbers 43,43. Many of these are in fact just different realizations of the same Calabi-Yau threefold. 


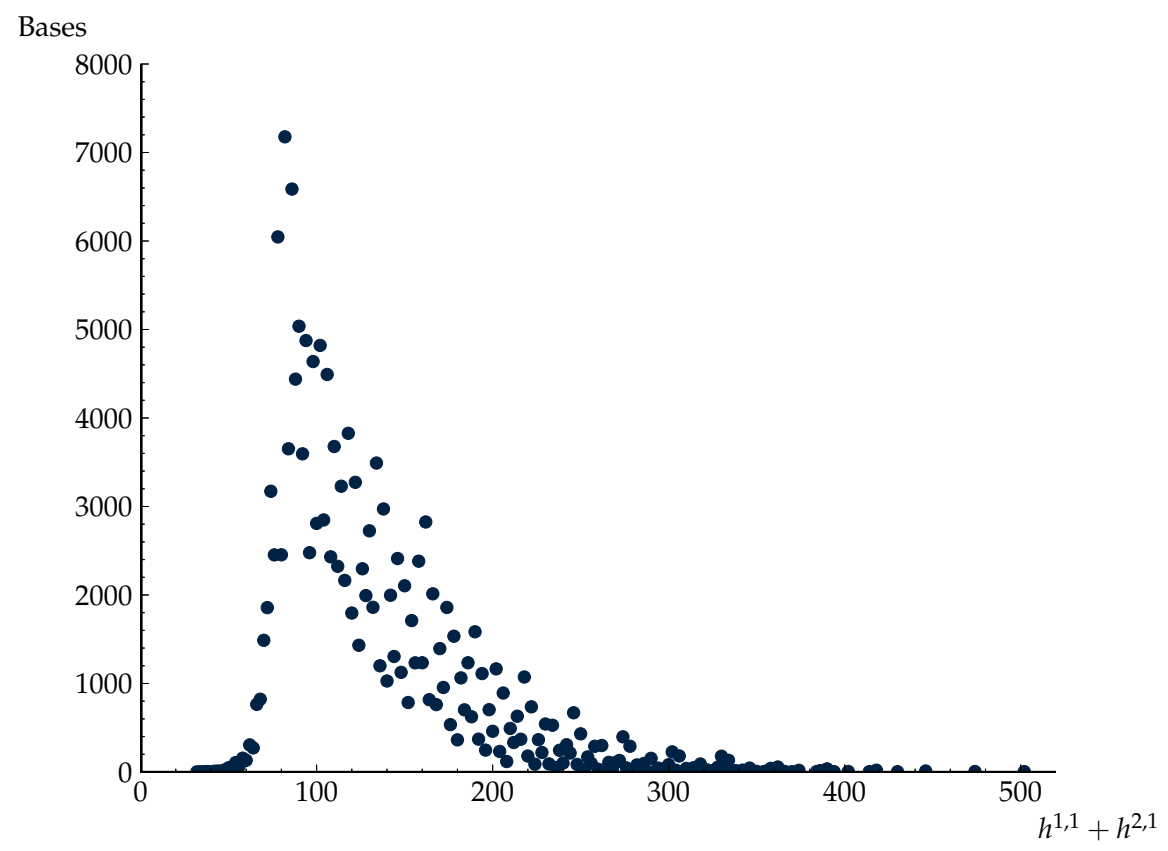

Figure 15. Distribution of threefolds coming from generic elliptic fibrations over $\mathbb{C}^{*}$-bases as a function of the sum of Hodge numbers.

One principal source of these kinds of redundancies arises from the appearance of clusters of -2 curves in the base that do not carry a gauge group. As discussed previously, such -2 curves indicate that a modulus of the geometry has been tuned. In general, we expect that any base containing a cluster of -2 curves is just be a special limit of another base without such clusters. Thus, we can consider the subset of $\mathbb{C}^{*}$-bases that do not contain any clusters of only -2 curves. This reduces the number of bases to 68,798 . A graph of the distribution of the numbers of bases without -2 clusters as a function of $T$ is given in figure 16 .

The removal of -2 clusters removes a great deal of redundancy in the list of threefolds. In particular, in the reduced set of bases the extreme jump in the distribution at $T=25$ goes away. This is associated with the removal of a large number of threefolds with Hodge numbers $(43,43)$. Looking at the detailed data shows that many of these $(43,43)$ models have a closely related structure. There are $1,575 \mathbb{C}^{*}$-bases that have the following features:

- Two $(-12)$ clusters and a gauge algebra $\mathfrak{e}_{8} \oplus \mathfrak{e}_{8}$

- $T=25$

- $h^{1,1}=43, h^{2,1}=43$.

In general, these bases are characterized by $\mathfrak{e}_{8}$ factors on the sections $\left(n_{0, \infty}=-12\right)$, and a set of chains containing various combinations of -2 curves. Indeed, it is clear that there are many ways to construct such $\mathbb{C}^{*}$-bases, by starting with a given $\mathbb{F}_{m}$ and 


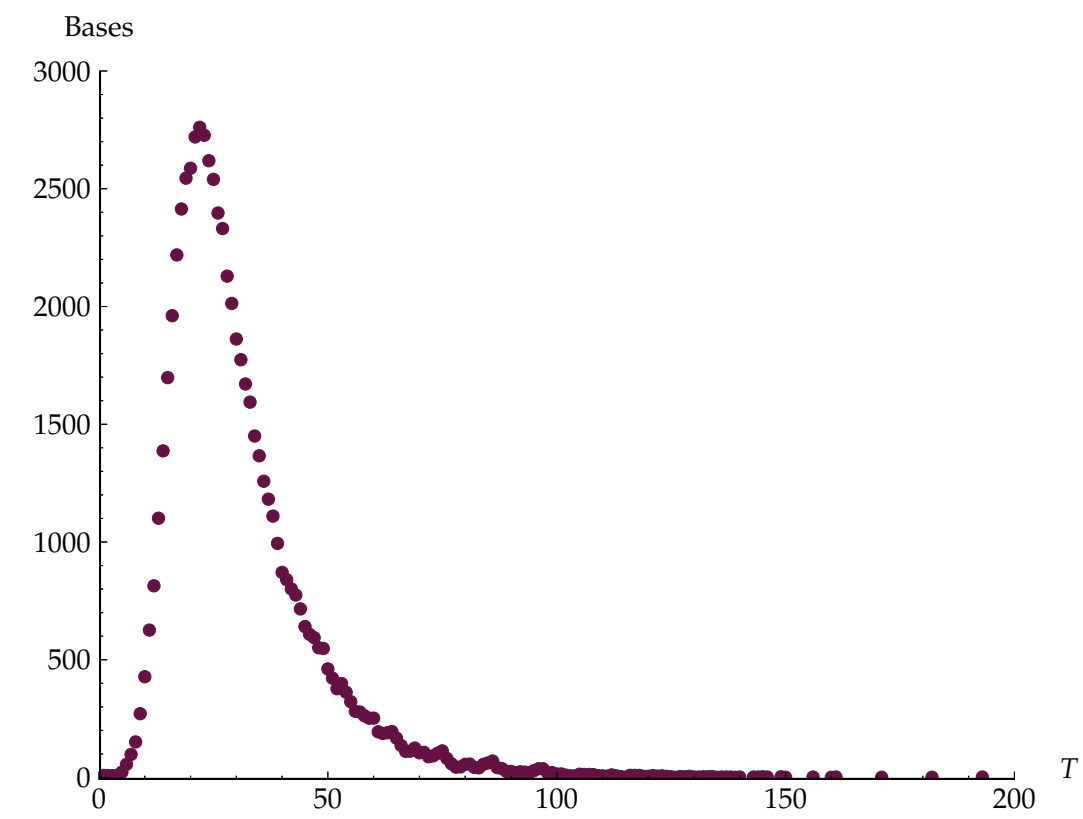

Figure 16. The number of $\mathbb{C}^{*}$-bases associated with different values of $T=h^{1,1}(B)-1$, when only bases without clusters of -2 curves not carried a gauge group are considered.

blowing up only points at $w=0, \infty$. Such $\mathbb{C}^{*}$-bases will always have chains of the form $(-1,-2,-2, \ldots,-2,-1)$. If precisely 24 points are blown up, giving the necessary factors on the sections, then any combination of chain lengths satisfying

$$
\sum_{i=1}^{N}\left(k_{i}-1\right)=24=T-1
$$

will have the desired properties. The number of such bases is just the number of ways of partitioning the 24 blow-ups into a sum of integers, $p(24)=1,575$, precisely the number of bases found with the above features. Of these partitionings, 13 have toric descriptions (partitions into one or two integers). All $1575 \mathbb{C}^{*}$-bases in this set can be understood as limit points of the same geometry, and are associated with the same smooth CalabiYau threefold. This Calabi-Yau threefold, which has been encountered previously in the literature (see e.g. [25]), seems to have a particularly high degree of symmetry and may be interesting for other reasons.

Although many of the bases with clusters of -2 curves have the same Hodge numbers as bases without such clusters, this is not universally true. There are also 3,788 $\mathbb{C}^{*}$ bases that have -2 clusters that have no corresponding $\mathbb{C}^{*}$-base without such clusters. An example is given by base $B$ with

$$
N=4, \quad n_{0}=-2, n_{\infty}=-4
$$

chain $1:(-2,-1,-4,-1,-3,-1)$

chains $2-4:(-1,-1)$ 
These bases may be limits of other base surfaces that are fine as complex surfaces but do not have a description as a $\mathbb{C}^{*}$-surface.

While as discussed above, in general bases with -2 clusters correspond to limits of bases without such clusters, and amounts to a redundancy for generic elliptic fibrations, keeping track of this information is often useful. In particular, Weierstrass models over a base with -2 clusters can be tuned to realize smooth resolved Calabi-Yau threefolds that cannot be realized by tuned Weierstrass models over the more generic bases without the -2 clusters. Thus, bases with -2 clusters must be considered separately in any systematic or complete analysis of a set of Calabi-Yau threefolds; examples of this arise in [47]. In terms of the physical F-theory models that can be constructed from these bases, the different -2 configurations and the distinct configurations of tuned gauge groups that can be realized over them correspond to distinct classes of $6 \mathrm{D}$ supergravity theories with distinct gauge group, matter, and dyonic string lattice structure. Thus, in a full consideration of $6 \mathrm{D}$ supergravity theories, it would be necessary to include all of the distinct base choices with Hodge numbers $(43,43)$ as initial points for tuned models with different sets of Hodge numbers.

\subsection{Calabi-Yau threefolds with new Hodge numbers}

Of the roughly $160,000 \mathbb{C}^{*}$-bases, we have found precisely 6 that give rise to generic elliptic fibrations with Hodge numbers that are not found in the Kreuzer-Skarke database. The simplest such base has Hodge numbers $h^{1,1}=56, h^{2,1}=2$, and the structure

$$
\begin{aligned}
N=3, & n_{0}=-5, n_{\infty}=-6, \\
\text { chain } 1: & (-1,-3,-1,-3,-1) \\
\text { chain 2: } & (-1,-3,-2,-1,-5,-1,-3,-1) \\
\text { chain } 3: & (-1,-3,-2,-2,-1,-6,-1,-3,-1)
\end{aligned}
$$

The other 5 bases that give new Calabi-Yau threefolds are listed in appendix B.

\subsection{Calabi-Yau threefolds with nontrivial Mordell-Weil rank}

There are $13 \mathbb{C}^{*}$-bases in which the rank of the Mordell-Weil group is nonzero. This is determined, as described above, by using the monomial count and the anomaly equation to independently determine $H_{\text {neutral }}$ and $H_{\text {neutral }}-V_{\text {abelian }}$ for each of the $\mathbb{C}^{*}$-bases. The bases where these two quantities differ are those for which the Mordell-Weil rank is nonzero. The elliptically fibered Calabi-Yau threefolds over these bases have multiple linearly independent sections in a group of rank $r$. This gives rise to $r$ abelian $\mathrm{U}(1)$ gauge fields in the corresponding 6D supergravity theory.

For these 13 bases, therefore, the Mordell-Weil rank is forced to be nontrivial even for a completely generic elliptic fibration. This is different from the situation for toric bases, where a complete analysis of all toric bases using the anomaly condition (4.4) confirmed that there are no toric bases over which the generic elliptic fibration has a nontrivial Mordell-Weil rank. 
An example of a base with nonzero Mordell-Weil rank is given by the following base with Hodge numbers $h^{1,1}=25, h^{2,1}=13$ :

$$
\begin{aligned}
N=3, & n_{0}=-1, n_{\infty}=-2, \\
\text { chain } 1: & (-2,-1,-2) \\
\text { chain } 2: & (-4,-1,-2,-2,-2) \\
\text { chain } 3: & (-4,-1,-2,-2,-2)
\end{aligned}
$$

The Hodge number $h^{1,1}$ can be computed from this base using (4.2), and $T=11$ from $(2.2)$, where $\mathcal{G}=\mathfrak{s o}(8) \oplus \mathfrak{s o}(8)$ from the two -4 curves, so $h^{1,1}(X)=T+2+8+r=21+r$, where $r$ is the rank of the Mordell-Weil group. Using the method of section 4.2, the number of Weierstrass monomials can be computed to be $W=7$. The dimension of the automorphism group is the generic $w_{\text {aut }}=1$, and $N_{-2}=9$ is the number of -2 curves, with $G_{1}=1$ as described at the end of section 4.2 since the -2 curves connected to $D_{\infty}$ have the $I V^{*}\left(\hat{E}_{6}\right)$ form from figure 13. It follows then from (4.12) that $H_{\text {neutral }}=14$, so $h^{2,1}=13$. Comparing with (4.4), we have

$$
V=H_{\text {neutral }}+29 T-273=60=56+r .
$$

Thus, this base has a generic elliptic fibration with Mordell-Weil rank $r=4$, and $h^{1,1}=25$. The 13 examples of bases with Mordell-Weil groups having nonzero rank are listed in appendix $\mathrm{C}$

The appearance of such bases, while perhaps surprising, is not completely unprecedented. It is known that the Shoen manifold, a class of elliptically fibered Calabi-Yau threefold constructed from a fiber product of rational elliptic surfaces, generically has Mordell-Weil rank 9 [48] (see e.g. [49] for a physics application of this). In fact, all the $\mathbb{C}^{*}$-bases we have identified that give Calabi-Yau threefolds with enhanced Mordell-Weil rank can be related to special limits and blow-ups of the Shoen manifold; ${ }^{6}$ this connection will be described in further detail elsewhere [50].

For the bases with nonzero Mordell-Weil rank, an explicit description of the Weierstrass model can be used to make the extra sections manifest. As an example, consider the base

$$
\begin{aligned}
N=4, & n_{0}=-6, n_{\infty}=-6, \\
\text { chain } 1: & (-1,-3,-1,-3,-1) \\
\text { chain } 2: & (-1,-3,-1,-3,-1) \\
\text { chain } 3: & (-1,-3,-1,-3,-1) \\
\text { chain } 4: & (-1,-3,-1,-3,-1)
\end{aligned}
$$

This base has $T=17, h^{1,1}=51, h^{2,1}=3$, and Mordell-Weil rank $r=4$. An explicit computation of the monomials in the Weierstrass model gives (placing the extra two fibers at $z=1,2)$

$$
\begin{aligned}
& f=A w^{4} z^{2}(z-1)^{2}(z-2)^{2} \\
& g=B w^{4} z^{6}(z-1)^{6}(z-2)^{6}+C w^{6} z^{3}(z-1)^{3}(z-2)^{3}+D w^{8},
\end{aligned}
$$

\footnotetext{
${ }^{6}$ Thanks to David Morrison for discussions on this point.
} 
where $A, B, C, D$ are free complex constants $(W=4)$. A nontrivial section can be associated with a factorization of the Weierstrass equation $y^{2}=x^{3}+f x+g$ into the form [33]

$$
(y-\alpha)(y+\alpha)=(x-\lambda)\left(x^{2}+\lambda x-\mu\right)
$$

where

$$
\begin{aligned}
& f=-\mu-\lambda^{2} \\
& g=\lambda \mu+\alpha^{2} .
\end{aligned}
$$

The Weierstrass coefficients from (4.18) can take this form if

$$
\begin{aligned}
& \lambda=a w^{2} z(z-1)(z-2) \\
& \mu=b w^{4} z^{2}(z-1)^{2}(z-2)^{2} \\
& \alpha=c w^{2} z^{3}(z-1)^{3}(z-2)^{3}+d w^{4}
\end{aligned}
$$

where $a, b, c, d$ satisfy

$$
\begin{aligned}
& A=-a^{2}-b \\
& B=c^{2} \\
& C=a b+2 c d \\
& D=d^{2}
\end{aligned}
$$

For given generic values of $A-D$ there are 12 solutions for $a$ - $d$. This can be seen by noting that the equations for $B, D$ each have two solutions for $c, d$, and the other two equations combine to form a cubic for $a$, which has 3 independent solutions. These twelve solutions represent 4 independent generators of the Mordell-Weil group. Solutions with $\alpha \rightarrow-\alpha$ correspond to sections $s,-s$ that add to 0 . The three solutions for the cubic also contain one linear dependence in the space of sections, so that the total number of independent sections is 4, matching the computed Mordell-Weil rank. A similar computation can be carried out for the other bases with enhanced Mordell-Weil rank, though the details are more complicated and computing the number of independent sections can be more difficult in other cases. A more detailed analysis of these models with enhanced Mordell-Weil rank is left to future work.

\section{Conclusions}

In this paper we have initiated a systematic study of a class of geometries for the bases of elliptically fibered Calabi-Yau threefolds that goes beyond the framework of toric geometry widely used in previous work. We have systematically constructed all smooth surfaces that admit a single $\mathbb{C}^{*}$ action and can arise as bases of a Calabi-Yau threefold, and we have analyzed the properties of these geometries. The 162,404 bases we have explicitly constructed include all $\mathbb{C}^{*}$-bases and also a more general class built from $\mathbb{C}^{*}$-bases containing $-9,-10$, and -11 curves on which points must be blown up to form -12 curves in bases that do not have a $\mathbb{C}^{*}$ action. 
The bases we have considered can be used for compactification of F-theory to six dimensions. We have found that the physical properties of the resulting six-dimensional supergravity theories are similar in nature and in distribution to compactifications on toric bases that were studied earlier. In particular, the $\mathbb{C}^{*}$-bases with relatively large numbers $T$ of tensor multiplets give theories with gauge algebras that are dominated by summands of the form $\mathfrak{e}_{8} \oplus \mathfrak{f}_{4} \oplus 2\left(\mathfrak{g}_{2} \oplus \mathfrak{s u}(2)\right)$, from the same types of chains that give this structure in the toric case. The largest value of $T$ for theories with $\mathbb{C}^{*}$-bases that are not actually toric is 171 , lower than the largest known value of $T=193$ that occurs for a toric base (in both cases, these are examples of bases containing -11 curves that must be blown up to give bases outside the $\mathbb{C}^{*} /$ toric framework). The overall "shield" structure of the set of Hodge numbers computed by Kreuzer and Skarke based on toric constructions, the boundary of this region explained in [12], and geometric patterns identified in $[12,51]$ are essentially unchanged with the addition of the large number of more general non-toric $\mathbb{C}^{*}$-base configurations. The absence of branching or loop structures in the $\mathbb{C}^{*}$-bases that make possible higher values of $T$ supports the conclusion that $T \leq 193$ is an absolute bound across all bases, as was argued heuristically in [12].

One interesting result of this analysis is that the number of additional bases added by extending the toric construction to include the more general class of bases admitting a $\mathbb{C}^{*}$ action does not produce a wild increase in the number of possible bases. As more points are blown up, the set of geometries is generally controlled by linear structures along the fiber, and the possibility of branching does not lead to a combinatorial explosion in intersecting divisor structures. This suggests that a systematic classification of all smooth bases for elliptically fibered Calabi-Yau threefolds, even without a single $\mathbb{C}^{*}$ action, may be computationally tractable. Such a classification would be quite challenging, since the intersection structure can become quite complicated - particularly for $T \geq 9$ where there can be an infinite number of distinct -1 curves on the bases, such as occurs for $\mathrm{dP}_{9}$. Nonetheless, by using the method of non-Higgsable clusters to characterize possible geometries it may be possible to get a handle on this problem. We leave this as a challenge for future work.

We have analyzed the Hodge structure of the smooth Calabi-Yau threefolds associated with generic elliptic fibrations over all the $\mathbb{C}^{*}$-bases. While the Hodge numbers are all within the boundaries defined by toric bases and the Kreuzer-Skarke "shield" shape, we have identified a number of Calabi-Yau threefolds with novel properties. There are 6 new threefolds that have Hodge numbers that we believe have not been previously identified, as well as 13 threefolds in which the Mordell-Weil rank of the elliptic fibration is nontrivial, corresponding to non-Higgsable $\mathrm{U}(1)$ factors in the corresponding $6 \mathrm{D}$ supergravity theory.

The basic approach to constructing a more general class of base manifolds for elliptic fibrations and F-theory using spaces with reduced numbers of $\mathbb{C}^{*}$ actions should be possible for base threefolds and fourfolds as well, giving rise to geometries for compactification of F-theory and M-theory to dimensions from 5 to 2 . The analysis of these constructions becomes significantly more complicated in lower dimensions, however. In particular, for F-theory compactifications to four dimensions on elliptically-fibered Calabi-Yau fourfolds with threefold bases, the analysis of bases with a $\mathbb{C}^{*} \times \mathbb{C}^{*}$ or $\mathbb{C}^{*}$ action would be much more difficult than for the class of $\mathbb{C}^{*}$-surfaces considered here. One difficulty in analyzing 
such constructions is the absence of a clear analogue to the anomaly condition (4.4) for 6D theories. The absence of such a condition makes it harder to compute the Mordell-Weil group in a general $4 \mathrm{D}$ case, and hence more difficult to precisely identify the Hodge numbers of the elliptically-fibered fourfold. While some progress was made in [52] in identifying 4D parallels to the $6 \mathrm{D}$ anomaly condition and related structures, more work is needed to have a systematic approach to analyzing F-theory base spaces with reduced toric symmetry in the $4 \mathrm{D}$ case.

\section{Acknowledgments}

We would like to thank Lara Anderson, Philip Candelas, Mboyo Esole, Ilarion Melnikov, David Morrison, Daniel Park, Tony Pantev, and Yinan Wang for helpful discussions. This research was supported by the DOE under contract \#DE-FC02-94ER40818, and was also supported in part by the National Science Foundation under Grant No. PHY-1066293. WT would like to thank the Aspen Center for Physics for hospitality during part of this work.

\section{A Rules for connecting clusters in $\mathbb{C}^{*}$-bases}

All configurations of non-Higgsable clusters (NHC's) that can be connected by a single -1 curve in a $\mathbb{C}^{*}$-base are listed in table 3 . Some examples of how some of these configurations can arise in $\mathbb{C}^{*}$-bases are given in figure 17 .

There are a few additional constraints not analyzed in [10], that arise from conditions on branching over a $-n$ curve with $n>1$; these additional constraints arise for similar reasons to those underlying the constraints on branching over a -1 curve. The additional constraints that must be imposed on such branchings are as follows:

- -3 curves:

A -3 curve that is connected to a -2 curve can be connected to at most one chain that begins $-1,-5, \ldots$.

- -5 curves:

A -5 curve can connect to at most two chains that begin $-1,-3,-2, \ldots$.

- -7 curves:

A -7 curve can connect to at most five chains that begin $-1,-2,-3, \ldots$.

- -8 curves:

A -8 curve can connect to at most four chains that begin $-1,-2,-3, \ldots$.

- -12 curves:

A -12 curve can connect to at most eight chains that begin $-1,-2,-2,-3, \ldots$. 


\begin{tabular}{|r|r|l|}
\hline Index $(n)$ & NHC & pairs $\{$ triplets [quadruplets] $\}$ \\
\hline 1 & $(-1 \dot{2})$ & 12 \\
2 & $(-\dot{8})$ & $9,12,13$ \\
3 & $(-\dot{7})$ & $9,12,13$ \\
4 & $(-\dot{6})$ & $7,9,11,12,13$ \\
5 & $(-\dot{5})$ & $7,8,9,10,11,12,13$ \\
6 & $(-\dot{4})$ & $6,7,8,9\{12\}, 10,11,12\{12,13\}, 13,14$ \\
7 & $(-\dot{3})$ & $7\{7,9,12\}, 8,9\{9,12,13\}, 10,11,12\{12,13\}, 13\{13\}, 14$ \\
8 & $(-\dot{3},-2)$ & $8,9\{9,12\}, 10,11,12\{12,13\}, 13,14$ \\
9 & $(-3,-\dot{2})$ & $9\{9,10,12[12], 13\}, 10\{12\}, 11,12\{12[12,13], 13\}, 13\{13\}, 14$ \\
10 & $(-\dot{3},-2,-2)$ & $10,11,12\{12,13\}, 13,14$ \\
11 & $(-3,-\dot{2},-2)$ & $12\{12,13\}, 13\{13\}$ \\
12 & $(-3,-2,-\dot{2})$ & $12\{12[12,13], 13[13]\}, 13\{13\}, 14$ \\
13 & $(-\dot{2},-3,-2)$ & $13\{13\}, 14$ \\
14 & $(-2,-\dot{3},-2)$ & \\
\hline
\end{tabular}

Table 3. Table of all ways in which connections between non-Higgsable clusters by (-1)-curves are allowed to appear in a $\mathbb{C}^{*}$-surface that can be used for an F-theory base. The dots specify the curves within each NHC that intersect with the (-1)-curve. The last column provides a complete list of the allowed pairs, triplets and quadruplets of NHCs that can be intersected by a single (-1)-curve in a valid F-theory base. No more than 4 NHCs can be connected by a single (-1) curve. Note also that any of the single NHCs or set of several NHCs that can be intersected by a single (-1)-curve can also be connected by the same (-1)-curve to sets of -2 curves, which do not carry a nonabelian gauge group. This table contains the subset of the data from an analogous table in [10] that is relevant for $\mathbb{C}^{*}$-bases. Note that combinations are listed only in increasing numerical order, so that a given combination only appears on the row associated with the lowest numbered NHC in the connected set.

As an example of how these constraints arise, consider the Zariski decomposition of $-4 K,-6 K$ for a sequence of rational curves $A, B, C, D$ of self-intersections $-5,-1,-3,-2$ with mutual intersections $A \cdot B=B \cdot C=C \cdot D$. From the relations $-K \cdot A=-3,-K \cdot C=$ $-1, \ldots$ we have

$$
\begin{aligned}
& -4 K=3 A+B+2 C+D+X \\
& -6 K=4 A+B+3 C+2 D+Y
\end{aligned}
$$

where $X, Y$ are residual effective divisors that have nonnegative intersection with each of the curves $A-D$. This Zariski decomposition is compatible with the $(3,4)$ vanishing on the -5 curve, etc., and implies that $(f, g)$ both vanish to order one on the curve $B$. Now, consider a configuration where the curve $A$ of self-intersection -5 is attached to three sequences $(B, C, D),\left(B^{\prime}, C^{\prime}, D^{\prime}\right),\left(B^{\prime \prime}, C^{\prime \prime}, D^{\prime \prime}\right)$ of curves of self-intersections $-1,-3,-2$. Then both $-4 K$ and $-6 K$ would have to contain all of $B, B^{\prime}, B^{\prime \prime}$ as components. Since, however, $\left(4 A+B+B^{\prime}+B^{\prime \prime}\right) \cdot A=-17$, while $-6 K \cdot A=-18$, the Zariski decomposition of $-6 K$ must contain at least $5 A$, so that $g$ must vanish to order 5 on $A$. This would mean, however, that $(f, g)$ would vanish to orders $(4,6)$ at the point $A \cap B$, which we do not 


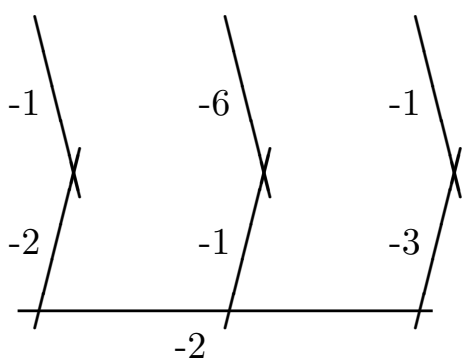

(a)

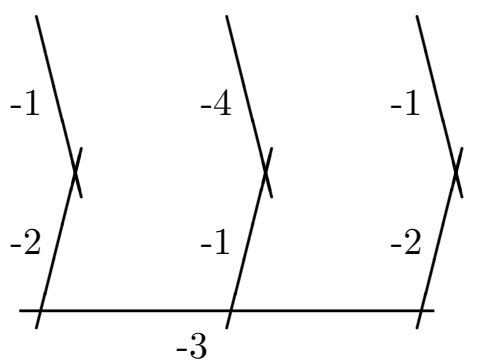

(b)

Figure 17. Examples of how clusters connected by -1 curves can arise at the branching points of the configuration of divisors in $\mathbb{C}^{*}$-bases.

allow. This demonstrates the condition stated above for branching on a -5 curve. Similar arguments can be used to show the other conditions.

The conditions on -3 and -5 curve branching rule out some additional candidates for $\mathbb{C}^{*}$-bases that are otherwise apparently acceptable. ${ }^{7}$

\section{B Bases giving Calabi-Yau threefolds with new Hodge numbers}

In addition to the example (4.14) given in section 4.5 , there are 5 other $\mathbb{C}^{*}$-bases over which the generic elliptic fibration has Hodge numbers not found in the Kreuzer-Skarke database: Hodge numbers $h^{1,1}=61, h^{2,1}=1$ :

$$
\begin{aligned}
N=3, & n_{0}=-5, n_{\infty}=-6, \\
\text { chain } 1: & (-1,-3,-1,-3,-1) \\
\text { chain } 2: & (-1,-3,-2,-2,-1,-6,-1,-3,-1) \\
\text { chain } 3: & (-1,-3,-2,-2,-1,-6,-1,-3,-1)
\end{aligned}
$$

Hodge numbers $h^{1,1}=62, h^{2,1}=2$ :

$$
\begin{aligned}
N=3, & n_{0}=-4, n_{\infty}=-8, \\
\text { chain } 1: & (-1,-4,-1,-2,-3,-2,-1) \\
\text { chain } 2: & (-1,-4,-1,-2,-3,-2,-1) \\
\text { chain } 3: & (-1,-4,-1,-2,-3,-2,-1)
\end{aligned}
$$

Hodge numbers $h^{1,1}=110, h^{2,1}=2$ :

$$
N=3, \quad n_{0}=-6, n_{\infty}=-12,
$$

chain 1 : $(-1,-3,-1,-3,-2,-2,-1)$

chain $2:(-1,-3,-1,-3,-2,-2,-1)$

chain $3:(-1,-3,-1,-6,-1,-3,-1,-6,-1,-3,-1,-5,-1,-3,-2,-2$,

$$
-1,-12,-1,-2,-2,-3,-1,-5,-1,-3,-2,-2,-1)
$$

\footnotetext{
${ }^{7}$ These additional rules were missed in an earlier version of this paper, which led to the appearance of four additional candidate $\mathbb{C}^{*}$-bases. Remarkably, these four all were in the class of bases with generic nonzero rank for the Mordell-Weil group. Thanks to D. Morrison, D. Park, and Y. Wang for discussions on related points.
} 
Hodge numbers $h^{1,1}=241, h^{2,1}=31$ :

$$
N=3, \quad n_{0}=-1, n_{\infty}=-5,
$$

chain $1:(-1,-1)$

chain $2:(-2,-1,-3,-1)$

chain $3:-12 / /-11 / /-12 / /-12 / /-12 / /-12 / /-12 / /-11,-1,-2,-2,-3,-1)$

where, as in [13], the notation $X / / Y$ denotes the sequence $X,-1,-2,-2,-3,-1,-5,-1$, $-3,-2,-2,-1, Y$.

Hodge numbers $h^{1,1}=252, h^{2,1}=30$ :

$$
N=3, \quad n_{0}=-1, n_{\infty}=-6,
$$

chain $1:(-1,-1)$

chain $2:(-2,-1,-3,-1)$

chain $3:-1,-12 / /-11 / /-12 / /-12 / /-12 / /-12 / /-12 / /-12 / /-1,-2,-2,-3$,

$$
-1,-5,-1,-3,-1)
$$

\section{Bases giving $\mathrm{U}(1)$ factors}

This appendix contains a list of the 13 bases over which a generic elliptic fibration has Mordell-Weil group of nonzero rank $r$. Note that two of these (numbers 4, 5) also appear in the list of Calabi-Yau's with new Hodge numbers.

$$
r=1: \quad N=3, n_{0}=-2, n_{\infty}=-3 ; \quad T=15 ; h^{1,1}=34, h^{2,1}=10
$$

chain $1:(-2,-1,-2)$

chain $2:(-2,-2,-1,-4,-1)$

chain $3:(-2,-2,-2,-2,-2-1,-8,-1,-2)$

$$
r=2: \quad N=3, n_{0}=-1, n_{\infty}=-2 ; \quad T=12 ; \quad h^{1,1}=24, h^{2,1}=12
$$

chain $1:(-2,-1,-2)$

chain $2:(-3,-1,-2,-2)$

chain $3:(-6,-1,-2,-2,-2,-2,-2)$

$$
r=2: \quad N=3, n_{0}=-2, n_{\infty}=-6 ; \quad T=18 ; h^{1,1}=46, h^{2,1}=10
$$

chain $1:(-2,-1,-3,-1)$

chain $2:(-2,-2,-2,-1,-6,-1,-3,-1)$

chain $3:(-2,-2,-2,-1,-6,-1,-3,-1)$ 


$$
r=2: \quad N=3, n_{0}=-5, n_{\infty}=-6 ; \quad T=21 ; \quad h^{1,1}=61, h^{2,1}=1
$$

chain 1 : $(-1,-3,-1,-3,-1)$

chain $2:(-1,-3,-2,-2,-1,-6,-1,-3,-1)$

chain $3:(-1,-3,-2,-2,-1,-6,-1,-3,-1)$

$$
r=3: \quad N=3, n_{0}=-4, n_{\infty}=-8 ; \quad T=19 ; \quad h^{1,1}=62, h^{2,1}=2
$$

chain $1:(-1,-4,-1,-2,-3,-2,-1)$

chain $2:(-1,-4,-1,-2,-3,-2,-1)$

chain $3:(-1,-4,-1,-2,-3,-2,-1)$

$$
r=4: \quad N=3, n_{0}=-1, n_{\infty}=-2 ; \quad T=11 ; h^{1,1}=25, h^{2,1}=13
$$

chain 1 : $(-2,-1,-2)$

chain $2:(-4,-1,-2,-2,-2)$

chain $3:(-4,-1,-2,-2,-2)$

$$
r=4: \quad N=3, n_{0}=-1, n_{\infty}=-5 \quad T=14 ; \quad h^{1,1}=40, h^{2,1}=4
$$

chain 1 : $(-2,-1,-3,-1)$

chain $2:(-4,-1,-2,-2,-3,-1)$

chain $3:(-4,-1,-2,-2,-3,-1)$

$$
r=4: \quad N=4, n_{0}=-6, n_{\infty}=-6 ; \quad T=17 ; \quad h^{1,1}=51, h^{2,1}=3
$$

chain $1:(-1,-3,-1,-3,-1)$

chain $2:(-1,-3,-1,-3,-1)$

chain $3:(-1,-3,-1,-3,-1)$

chain $4:(-1,-3,-1,-3,-1)$

$$
r=4: \quad N=3, n_{0}=-2, n_{\infty}=-4 ; \quad T=13 ; h^{1,1}=35, h^{2,1}=11
$$

chain $1:(-2,-2,-1,-4,-1)$

chain $2:(-2,-2,-1,-4,-1)$

chain $3:(-2,-2,-1,-4,-1)$

$$
r=5: \quad N=3, n_{0}=-1, n_{\infty}=-8 ; \quad T=16 ; h^{1,1}=51, h^{2,1}=3
$$

chain $1:(-3,-1,-2,-3,-2,-1)$

chain $2:(-3,-1,-2,-3,-2,-1)$

chain $3:(-3,-1,-2,-3,-2,-1)$ 


$$
r=6: \quad N=3, n_{0}=-1, n_{\infty}=-2 ; \quad T=10 ; \quad h^{1,1}=24, h^{2,1}=12
$$

chain $1:(-3,-1,-2,-2)$

chain $2:(-3,-1,-2,-2)$

chain $3: \quad(-3,-1,-2,-2)$

$$
r=6: \quad N=4, n_{0}=-2, n_{\infty}=-6 ; \quad T=13 ; \quad h^{1,1}=35, h^{2,1}=11
$$

chain $1:(-2,-1,-3,-1)$

chain $2:(-2,-1,-3,-1)$

chain $3:(-2,-1,-3,-1)$

chain $4:(-2,-1,-3,-1)$

$$
r=8: \quad N=4, n_{0}=-2, n_{\infty}=-2 ; \quad T=9 ; \quad h^{1,1}=19, h^{2,1}=19
$$

chain $1:(-2,-1,-2)$

chain $2:(-2,-1,-2)$

chain $3:(-2,-1,-2)$

chain $4:(-2,-1,-2)$

Note that this last example is a special limit of $d P_{9}$ with two -2 clusters of type $I_{0}^{*}\left(\hat{D}_{4}\right)$.

Open Access. This article is distributed under the terms of the Creative Commons Attribution License (CC-BY 4.0), which permits any use, distribution and reproduction in any medium, provided the original author(s) and source are credited.

\section{References}

[1] M.B. Green, J.H. Schwarz and E. Witten, Superstring Theory, Vols. 1, 2 Cambridge University Press, Cambridge U.K. (1987).

[2] J. Polchinski, String theory, Cambridge University Press, Cambridge U.K. (1998).

[3] C. Vafa, Evidence for F-theory, Nucl. Phys. B 469 (1996) 403 [hep-th/9602022] [INSPIRE].

[4] D.R. Morrison and C. Vafa, Compactifications of F-theory on Calabi-Yau threefolds. 1, Nucl. Phys. B 473 (1996) 74 [hep-th/9602114] [INSPIRE].

[5] D.R. Morrison and C. Vafa, Compactifications of F-theory on Calabi-Yau threefolds. 2., Nucl. Phys. B 476 (1996) 437 [hep-th/9603161] [InSPIRE].

[6] R. Davies, The Expanding Zoo of Calabi-Yau Threefolds, Adv. High Energy Phys. 2011 (2011) 901898 [arXiv:1103.3156] [INSPIRE].

[7] W. Fulton, Introduction to Toric Varieties, Annals of Mathematics Study 131, Princeton University Press, Princeton U.K. (1993).

[8] V. Batyrev, Variations of the mixed Hodge structure of affine hypersurfaces in algebraic tori, Duke Math. J. 69 (1993) 349. 
[9] M. Kreuzer and H. Skarke, Complete classification of reflexive polyhedra in four-dimensions, Adv. Theor. Math. Phys. 4 (2002) 1209 [hep-th/0002240] [INSPIRE].

[10] D.R. Morrison and W. Taylor, Classifying bases for 6D F-theory models, Central Eur. J. Phys. 10 (2012) 1072 [arXiv:1201.1943] [INSPIRE].

[11] K. Altmann, N.O. Ilten, L. Petersen, H. Süss and R. Vollmert, The geometry of T-varieties, arXiv: 1102.5760 [INSPIRE].

[12] D.R. Morrison and W. Taylor, Toric bases for 6D F-theory models, Fortsch. Phys. 60 (2012) 1187 [arXiv: 1204. 0283] [INSPIRE].

[13] W. Taylor, On the Hodge structure of elliptically fibered Calabi-Yau threefolds, JHEP 08 (2012) 032 [arXiv: 1205.0952] [INSPIRE].

[14] D.R. Morrison, TASI lectures on compactification and duality, hep-th/0411120 [INSPIRE].

[15] F. Denef, Les Houches Lectures on Constructing String Vacua, arXiv:0803.1194 [INSPIRE].

[16] W. Taylor, TASI Lectures on Supergravity and String Vacua in Various Dimensions, arXiv:1104.2051 [INSPIRE].

[17] F. Bonetti and T.W. Grimm, Six-dimensional $(1,0)$ effective action of F-theory via M-theory on Calabi-Yau threefolds, JHEP 05 (2012) 019 [arXiv:1112.1082] [INSPIRE].

[18] V. Kumar, D.S. Park and W. Taylor, 6D supergravity without tensor multiplets, JHEP 04 (2011) 080 [arXiv: 1011.0726] [INSPIRE].

[19] V. Braun, Toric Elliptic Fibrations and F-theory Compactifications, JHEP 01 (2013) 016 [arXiv:1110.4883] [INSPIRE].

[20] W.P. Barth, K. Hulek, C.A.M. Peters and A. Van de Ven, Compact complex surfaces, Springer, Germany (2004).

[21] M. Reid, Chapters on algebraic surfaces, Complex algebraic geometry, Park City UT, U.S.A. (1993), IAS/Park City Math. Ser. 3 (1997) 3 [alg-geom/9602006].

[22] A. Grassi, On minimal models of elliptic threefolds, Math. Ann. 290 (1991) 287.

[23] M. Gross, A finiteness theorem for elliptic Calabi-Yau threefolds, Duke Math. J. 74 (1994) 271.

[24] V. Kumar, D.R. Morrison and W. Taylor, Global aspects of the space of $6 D N=1$ supergravities, JHEP 11 (2010) 118 [arXiv:1008.1062] [INSPIRE].

[25] P. Candelas, E. Perevalov and G. Rajesh, Toric geometry and enhanced gauge symmetry of F-theory/heterotic vacua, Nucl. Phys. B 507 (1997) 445 [hep-th/9704097] [InSPIRE].

[26] P.S. Aspinwall and D.R. Morrison, Point - like instantons on K3 orbifolds, Nucl. Phys. B 503 (1997) 533 [hep-th/9705104] [INSPIRE].

[27] J.J. Heckman, D.R. Morrison and C. Vafa, On the Classification of 6D SCFTs and Generalized ADE Orbifolds, JHEP 05 (2014) 028 [arXiv: 1312.5746] [INSPIRE].

[28] R. Wazir, Arithmetic on elliptic threefolds, Compos. Math. 140 (2004) 567.

[29] K. Hulek and R. Kloosterman, Calculating the Mordell-Weil rank of elliptic threefolds and the cohomology of singular hypersurfaces, arXiv:0806.2025.

[30] T.W. Grimm and T. Weigand, On Abelian Gauge Symmetries and Proton Decay in Global F-theory GUTs, Phys. Rev. D 82 (2010) 086009 [arXiv: 1006. 0226] [INSPIRE].

[31] D.S. Park and W. Taylor, Constraints on 6D Supergravity Theories with Abelian Gauge Symmetry, JHEP 01 (2012) 141 [arXiv:1110.5916] [INSPIRE]. 
[32] D.S. Park, Anomaly Equations and Intersection Theory, JHEP 01 (2012) 093 [arXiv:1111.2351] [INSPIRE].

[33] D.R. Morrison and D.S. Park, F-Theory and the Mordell-Weil Group of Elliptically-Fibered Calabi-Yau Threefolds, JHEP 10 (2012) 128 [arXiv:1208.2695] [INSPIRE].

[34] C. Mayrhofer, E. Palti and T. Weigand, U(1) symmetries in F-theory GUTs with multiple sections, JHEP 03 (2013) 098 [arXiv:1211.6742] [INSPIRE].

[35] V. Braun, T.W. Grimm and J. Keitel, New Global F-theory GUTs with U(1) symmetries, JHEP 09 (2013) 154 [arXiv: 1302.1854] [INSPIRE].

[36] J. Borchmann, C. Mayrhofer, E. Palti and T. Weigand, Elliptic fibrations for $S U(5) \times U(1) \times U(1)$ F-theory vacua, Phys. Rev. D 88 (2013) 046005 [arXiv:1303.5054] [INSPIRE].

[37] M. Cvetič, D. Klevers and H. Piragua, F-Theory Compactifications with Multiple U(1)-Factors: Constructing Elliptic Fibrations with Rational Sections, JHEP 06 (2013) 067 [arXiv: 1303.6970] [INSPIRE].

[38] M. Cvetic, D. Klevers and H. Piragua, F-Theory Compactifications with Multiple U(1)-Factors: Addendum, JHEP 12 (2013) 056 [arXiv: 1307.6425] [INSPIRE].

[39] V. Braun, T.W. Grimm and J. Keitel, Geometric Engineering in Toric F-theory and GUTs with U(1) Gauge Factors, JHEP 12 (2013) 069 [arXiv:1306.0577] [INSPIRE].

[40] J. Borchmann, C. Mayrhofer, E. Palti and T. Weigand, SU(5) Tops with Multiple U(1)s in F-theory, Nucl. Phys. B $\mathbf{8 8 2}$ (2014) 1 [arXiv:1307.2902] [InSPIRE].

[41] M. Cvetič, D. Klevers, H. Piragua and P. Song, Elliptic fibrations with rank three Mordell-Weil group: F-theory with $U(1) \times U(1) \times U(1)$ gauge symmetry, JHEP 03 (2014) 021 [arXiv: 1310.0463] [INSPIRE].

[42] A.P. Braun, A. Collinucci and R. Valandro, The fate of U(1)'s at strong coupling in F-theory, JHEP 07 (2014) 028 [arXiv: 1402.4054] [INSPIRE].

[43] M.R. Douglas, D.S. Park and C. Schnell, The Cremmer-Scherk Mechanism in F-theory Compactifications on K3 Manifolds, JHEP 05 (2014) 135 [arXiv:1403.1595] [INSPIRE].

[44] D.R. Morrison and W. Taylor, Sections, multisections and U(1) fields in F-theory, arXiv: 1404.1527 [INSPIRE].

[45] M.B. Green, J.H. Schwarz and P.C. West, Anomaly Free Chiral Theories in Six-Dimensions, Nucl. Phys. B 254 (1985) 327 [InSPIRE].

[46] A. Sagnotti, A Note on the Green-Schwarz mechanism in open string theories, Phys. Lett. B 294 (1992) 196 [hep-th/9210127] [inSPIRE].

[47] S. Johnson and W. Taylor, Calabi-Yau threefolds with large $h^{2,1}$, to appear.

[48] K. Oguiso and T. Shioda, The Mordell-Weil lattice of a rational elliptic surface, Comment. Math. Univ. St. Paul. 40 (1991) 83.

[49] V. Braun, B.A. Ovrut, T. Pantev and R. Reinbacher, Elliptic Calabi-Yau threefolds with $Z(3) \times Z(3)$ Wilson lines, JHEP 12 (2004) 062 [hep-th/0410055] [INSPIRE].

[50] D.R. Morrison, D. Park and W. Taylor, to appear.

[51] P. Candelas, A. Constantin and H. Skarke, An Abundance of K3 Fibrations from Polyhedra with Interchangeable Parts, Commun. Math. Phys. 324 (2013) 937 [arXiv:1207.4792] [INSPIRE]. 
[52] T.W. Grimm and W. Taylor, Structure in $6 D$ and $4 D N=1$ supergravity theories from F-theory, JHEP 10 (2012) 105 [arXiv: 1204.3092] [INSPIRE]. 